\title{
Seeds of large-scale anisotropy in string cosmology
}

\author{
R. Durrer, ${ }^{1}$ M. Gasperini, ${ }^{2}$ M. Sakellariadou, ${ }^{1}$ and G. Veneziano ${ }^{3}$ \\ ${ }^{1}$ Département de Physique Théorique, Université de Genève, 24 quai E. Ansermet, CH-1211 Geneva, Switzerland \\ ${ }^{2}$ Dipartimento di Fisica Teorica, Università di Torino, Via P. Giuria 1, 10125 Turin, Italy \\ ${ }^{3}$ Theory Division, CERN, CH-1211 Geneva 23, Switzerland
}

(Received 23 April 1998; published 22 January 1999)

\begin{abstract}
Pre-big-bang cosmology predicts tiny first-order dilaton and metric perturbations at very large scales. Here we discuss the possibility that other, more copiously generated, perturbations may act, at second order, as scalar seeds of large-scale structure and CMB anisotropies. We study, in particular, the cases of electromagnetic and axionic seeds. We compute the stochastic fluctuations of their energy-momentum tensor and determine the resulting contributions to the multipole expansion of the temperature anisotropy. In the axion case it is possible to obtain a flat or slightly tilted blue spectrum that fits present data consistently, both for massless and for massive (but very light) axions. [S0556-2821(99)03102-1]
\end{abstract}

PACS number(s): 98.80.Cq

\section{INTRODUCTION}

String theory has recently motivated the study of a cosmological scenario in which the universe, starting from the string perturbative vacuum, evolves through an early inflationary "pre-big-bang" phase [1], until a transition to the radiation-dominated, decelerated evolution occurs.

In spite of some attractive aspects of the pre-big-bang picture, such as the underlying duality symmetry [2], which naturally selects perturbative initial conditions and automatically leads to inflation $[1,3]$, it is fair to say that such a cosmological scenario is far from being understood in all of its aspects. In particular, on the more theoretical side, one is lacking a complete and consistent description of the highcurvature, strong coupling regime, where the transition from the pre- to the post-big-bang era is expected to take place [4]. Furthermore, opinions vary $[3,5]$ as to whether or not the pre-big-bang scenario needs a large amount of fine-tuning. On a more phenomenological side, the main outstanding problem is to reproduce the observed amplitude and slope of the large-scale temperature anisotropy [6] and of large-scale density perturbations. The difficulty is that, unlike in the more conventional (de Sitter-like) inflationary picture, the amplification of scalar and tensor metric perturbations here leads to primordial spectra that grow with frequency [7], and whose energy density is normalized to an almost critical value at some short scale [8] (typically the GHz); in this way, too little power is left at scales that are relevant for anisotropies in the cosmic microwave background (CMB) [6] or to the problem of large-scale structure (unless the highcurvature phase is long enough and characterized by an almost constant dilaton field [9]).

In this paper we address this problem and we discuss a possible solution, based on the contribution of "seeds" [10] to density fluctuations and to the anisotropy of the CMB radiation. The seeds are produced, in our context, by the amplification of quantum fluctuations of some other fields, which are present in string theory, but are not part of the homogeneous background whose perturbations we wish to study.

We shall consider two examples, in which the seed inho- mogeneity spectrum is due, respectively, to vacuum fluctuations of the electromagnetic (EM) [11] and of the [KalbRamond (KR)] axion (AX) [12] field. Both cases are typical of string cosmology, since no inhomogeneity is produced, in either case, in a conventional scenario based on Einstein's equations, without axion and dilaton. The spectra of EM and AX perturbations can be much flatter than those of scalar and tensor perturbations of the metric and of the dilaton field.

The idea of using the EM fluctuations as seeds was already discussed in a previous paper [13], using however the perfect fluid approximation for the EM stress tensor. Here we will compute the scalar components of the energymomentum-tensor fluctuations due to the EM and AX seeds including an important anisotropic stress term, and will relate them to the primordial spectral energy distributions. When these seed inhomogeneities are inserted in the perturbed Einstein equations they generate scalar-metric fluctuations which are largely controlled, for seeds with small enough anisotropic stresses, on super-horizon scales, by the so-called compensation mechanism [14]. Finally, scalar-metric perturbations can be converted in a standard manner into temperature fluctuations $\Delta T / T$ via the Sachs-Wolfe effect [15]. We will discuss whether the metric perturbation spectrum induced by seeds can be flat enough to match present observations, consistently with the Cosmic Background Explorer (COBE) normalization of the amplitude on large scales, and with the high-frequency normalization of the primordial seed spectrum.

It should be stressed that, in our model, the axion is not to be identified with dark matter. The KR axions are treated here as "seeds," i.e. as inhomogeneous perturbations of a background which is not axion-dominated, so that our mechanism of anysotropy production is different from that of previous computation of isocurvature [16] and adiabatic [17] axion perturbations.

The paper aims at being rather self-contained and readable by non-specialists in string and/or cosmological perturbation theory, and is organized as follows. In Sec. II we set up the relevant equations needed to study super-horizon perturbations in the presence of seeds, and give their generic solution for seeds with "small" or "large" anisotropic 
stresses. We also discuss the way the perturbations enter the multipole expansion of $\Delta T / T$ via the Sachs-Wolfe effect. In Sec. III, after recalling known results about scalar, tensor, electromagnetic and axion perturbations in the pre-big-bang scenario, we estimate the contribution of the two latter sources to the fluctuations of the energy-momentum tensor, including the case of massive axions. In Sec. IV we combine the results of the previous two sections and compute the contribution of EM and $\mathrm{AX}$ seeds to $\Delta T / T$. Using COBE data, we finally discuss, in the various cases, whether the seed mechanism alone is able to give a satisfactory explanation of large-scale temperature anisotropies. Section V contains our conclusions. Some technical details are relegated to the three Appendixes.

Notation. The Friedmann metric is given by $a^{2}\left(-d \eta^{2}\right.$ $\left.+\gamma_{i j} d x^{i} d x^{j}\right)$, where $a$ denotes the scale factor and $\eta$ is conformal time. Spatial indices, 1,2,3 are denoted by latin letters while spacetime indices, $0,1,2,3$ are denoted by greek letters. A dot denotes derivative with respect to $\eta$.

\section{LARGE-SCALE PERTURBATIONS IN THE PRESENCE OF SEEDS}

Before calculating CMB anisotropies for specific examples in the context of string cosmology, we derive a general formula for large-scale CMB anisotropies in models with seed perturbations.

\section{A. Cosmological perturbation theory with seeds}

In this subsection we give a brief reminder of gaugeinvariant perturbation theory with seeds. More details can be found in Refs. $[10,18]$. By seeds we mean an inhomogeneously distributed form of energy, which contributes only a small fraction to the total energy density of the universe and can thus be considered as a perturbation. Furthermore, we consider seeds that interact only gravitationally with the cosmic fluid.

We restrict our discussion to scalar perturbations, which are of primary interest here. The corresponding equations for vector and tensor perturbations can be found in [18]. The metric of a perturbed Friedmann universe is

$$
g_{\mu \nu}=g_{\mu \nu}^{(0)}+a^{2} h_{\mu \nu},
$$

where $g^{(0)}$ denotes the unperturbed metric

$$
g_{\mu \nu}^{(0)} d x^{\mu} d x^{\nu}=a^{2}(\eta)\left(-d \eta^{2}+\gamma_{i j} d x^{i} d x^{j}\right) .
$$

Here $a$ is the scale factor, $\eta$ denotes conformal time and $\gamma$ represents a metric of constant curvature $K= \pm 1,0$. Since we will be interested in a Friedmann universe that has undergone substantial inflation, we neglect $K$ in the sequel, setting $\gamma_{i j}=\delta_{i j}$.

For scalar perturbations, a Fourier component of $h_{\mu \nu}$ with wave vector $\mathbf{k}$ can be parametrized by 4 scalar functions $A$, $B, H_{L}$ and $H_{T}$, defined by

$$
\begin{aligned}
h(k)= & h_{\mu \nu}(k) d x^{\mu} d x^{\nu} \\
= & -2 A(k)(d \eta)^{2}-2 i \frac{k_{j}}{k} B(k) d \eta d x^{j} \\
& +2\left[H_{L}(k)+\frac{1}{3} H_{T}(k)\right] \delta_{l j} d x^{l} d x^{j} \\
& -2 \frac{k_{l} k_{j}}{k^{2}} H_{T}(k) d x^{l} d x^{j} .
\end{aligned}
$$

These four functions are gauge-dependent, i.e., they depend on the choice of coordinates. In order to define gaugeindependent metric variables, we first make use of two geometric quantities: the spatial part of the scalar curvature of the perturbed metric, $\delta R$, and the shear (traceless) part of the extrinsic curvature, $K^{(\text {aniso })}$. An elementary calculation gives [18]

$$
\begin{gathered}
\delta R=4 k^{2} a^{-2} \mathcal{R}, \quad \mathcal{R}=H_{L}+\frac{1}{3} H_{T}, \\
K_{i j}^{(a n i s o)}=a k\left(\frac{k_{i} k_{j}}{k^{2}}-\frac{1}{3} \delta_{i j}\right) \sigma, \quad \sigma=\dot{H}_{T} / k-B .
\end{gathered}
$$

Studying the gauge transformation properties of $A, \mathcal{R}$, and $\sigma$, one easily finds that the following variables, called the (Fourier components of the) Bardeen potentials, are gauge invariant (see $[19,20])$ :

$$
\begin{aligned}
& \Phi=\mathcal{R}-(\dot{a} / a) k^{-1} \sigma, \\
& \Psi=A-(\dot{a} / a) k^{-1} \sigma-k^{-1} \dot{\sigma} .
\end{aligned}
$$

(Note that, throughout this paper, we shall always express the Bardeen potentials in momentum space, even without indicating their $k$ dependence explicitly.)

Next, we discuss the perturbations of the energymomentum tensor. Let us define the perturbed energy density $\rho^{(p e r t)}$ and 4-velocity field $u$ as the time-like eigenvalue and eigenvector of the energy-momentum tensor:

$$
T_{\mu}{ }^{\nu} u^{\mu}=-\rho^{(p e r t)} u^{\nu}, \quad u^{2}=-1 .
$$

The Fourier components of the perturbations in the density and velocity field are determined by

$$
\begin{aligned}
\rho^{(\text {pert })} & =\rho(1+\delta), \\
u^{0} & =(1-A), \quad \frac{u^{j}}{u^{0}}=-i \frac{k^{j}}{k} v,
\end{aligned}
$$

where $\rho$ denotes the unperturbed background density. The temporal component $u^{0}$ is fixed by the normalization condition. We project the stress tensor onto the 3-space orthogonal to $u$ :

$$
\tau_{\mu \nu}=P_{\mu}^{\rho} P_{\nu}^{\sigma} T_{\rho \sigma}, \quad P_{\mu \nu} \equiv g_{\mu \nu}+u_{\mu} u_{\nu},
$$

and define the scalar perturbations of $\tau$ by 


$$
\tau_{i}^{j}=p\left[\left(1+\pi_{L}+\frac{1}{3} \pi_{T}\right) \delta_{i}^{j}-\frac{k_{i} k^{j}}{k^{2}} \pi_{T}\right] .
$$

The variable $\pi_{L}$ describes the pressure perturbation, $\pi_{T}$ is the potential of the anisotropic stresses and $p$ is the unperturbed background pressure. Studying the behavior of the quantities $\delta, v, \pi_{L}$ and $\pi_{T}$ under gauge transformations [21], one finds the gauge-invariant variables:

$$
\begin{gathered}
\Pi=\pi_{T}, \quad \Gamma=\pi_{L}-\left(c_{s}^{2} / w\right) \delta, \quad V=v-k^{-1} \dot{H}_{T}, \\
D=\delta+3(1+w)(\dot{a} / a) k^{-1}(V+\sigma), \quad D_{g}=\delta+3(1+w) \mathcal{R} .
\end{gathered}
$$

Here $\Pi$ is the anisotropic stress potential, $\Gamma$ is the entropy perturbation, $V$ is the peculiar velocity potential, $D$ and $D_{g}$ are different choices for a gauge-invariant density perturbation variable (for a physical interpretation of these variables, see $[20,21])$. Finally, $w=p / \rho$ denotes the enthalpy and $c_{s}^{2}$ $=\dot{p} / \dot{\rho}$ stands for the adiabatic speed of sound. In this paper we shall limit ourselves to adiabatic perturbations $(\Gamma=0)$.

The perturbation of Einstein's equations and of energymomentum conservation can be expressed in terms of these gauge-invariant variables (a derivation can be found in $[20,21])$. We obtain two constraint equations,

$$
\begin{gathered}
4 \pi G a^{2} \rho D=k^{2} \Phi, \\
4 \pi G a^{2}(\rho+p) V=k[(\dot{a} / a) \Psi-\dot{\Phi}],
\end{gathered}
$$

two dynamical equations,

$$
\begin{gathered}
-8 \pi G a^{2} p \Pi=k^{2}(\Phi+\Psi) \\
8 \pi G a^{2} p\left[\Gamma+\left(c_{s}^{2} / w\right) D_{g}+(2 / 3) k^{2} \Pi\right] \\
=\frac{\dot{a}}{a}\left\{\dot{\Psi}-\left[a^{-1}\left(\frac{a^{2} \Phi}{\dot{a}}\right)^{\bullet}\right]\right\} \\
+\left[\frac{2}{a}\left(\frac{\dot{a}}{a^{2}}\right)^{\bullet}+3\left(\frac{\dot{a}}{a^{2}}\right)^{2}\right]\left[\Psi-a^{-1}\left(\frac{a^{2} \Phi}{\dot{a}}\right)^{\bullet}\right]
\end{gathered}
$$

and two conservation equations:

$$
\begin{aligned}
\dot{D}_{\alpha}-3 w_{\alpha}(\dot{a} / a) D_{\alpha} & \\
= & -k\left[\left(1+w_{\alpha}\right) V_{\alpha}+2(\dot{a} / a) w_{\alpha} k^{-1} \Pi_{\alpha}\right] \\
& +3\left(1+w_{\alpha}\right) 4 \pi G a^{2}(\rho+p)\left(V-V_{\alpha}\right), \\
\dot{V}_{\alpha}+(\dot{a} / a) V_{\alpha}= & \frac{c_{\alpha}^{2}}{1+w_{\alpha}} k D_{\alpha}+\frac{w_{\alpha}}{1+w_{\alpha}} k \Gamma_{\alpha} \\
& +k \Psi-\frac{2 w_{\alpha}}{3\left(1+w_{\alpha}\right)} k \Pi_{\alpha} .
\end{aligned}
$$

The above conservation equations hold for any component $\alpha$ of the fluid stress-energy tensor which interacts with the other components of the cosmic fluid only gravitationally. The variables $c_{\alpha}$ and $w_{\alpha}$ denote the adiabatic speed of sound and the enthalpy of the fluid component, respectively. The total perturbations are defined as the sums:

$$
\rho D=\sum_{\alpha} \rho_{\alpha} D_{\alpha}, \quad(\rho+p) V=\sum_{\alpha}\left(\rho_{\alpha}+p_{\alpha}\right) V_{\alpha} \text {, etc. }
$$

For interacting matter, the corresponding equations can be found in [20].

In order to complete the above analysis we also need equations of state for the matter sources, which relate for instance $\Gamma$ and $\Pi$ to $D$ and $V$. Due to the Bianchi identities, the conservation equations for the total cosmic fluid follow from the field equations (2.14)-(2.17). Thus, we need not make explicit use of both dynamical equations, but we can use, say, Eq. (2.16) and one of the conservation equations (2.18), (2.19) for the total fluid.

We now add to the perturbation equations an inhomogeneous energy-momentum distribution, $T_{\mu \nu}^{(s)}$, generated by seed fields that do not interact with the cosmic fluid other than gravitationally.

Since, by definition, seeds do not contribute as sources of the homogeneous background, the energy-momentum tensor $T_{\mu \nu}^{(s)}$ is gauge-invariant by itself [22], and can be calculated by solving the field equations for the seeds in the unperturbed background geometry. Let us assume that we can express the Fourier components of $T_{\mu \nu}^{(s)}$ in terms of four scalar "seed-functions" $f_{\rho}, f_{p}, f_{v}$ and $f_{\pi}$ (we just neglect vector and tensor contributions; since they are decoupled from density perturbations, in the linear approximation, this will not affect our results for scalar perturbations):

$$
\begin{aligned}
T_{00}^{(s)}(\mathbf{k}, \eta) & =a^{2} \rho^{(s)}=M^{2} f_{\rho}(\mathbf{k}, \eta) \\
T_{j 0}^{(s)}(\mathbf{k}, \eta) & =-i \frac{k_{j}}{k} a^{2} v^{(s)}=-i M^{2} k_{j} f_{v}(\mathbf{k}, \eta) \\
T_{i j}^{(s)}(\mathbf{k}, \eta) & =a^{2}\left[\left(p^{(s)}+\frac{1}{3} \Pi^{(s)}\right) \gamma_{i j}-\frac{k_{i} k_{j}}{k^{2}} \Pi^{(s)}\right] \\
& =M^{2}\left[\left(f_{p}(\mathbf{k}, \eta)+\frac{k^{2}}{3} f_{\pi}(\mathbf{k}, \eta)\right) \gamma_{i j}-k_{i} k_{j} f_{\pi}(\mathbf{k}, \eta)\right]
\end{aligned}
$$

Note that $f_{\rho}$ and $f_{p}$ have dimension $l^{-2}$, while $f_{v}$ has dimension $l^{-1}$ and $f_{\pi}$ is dimensionless. Here $M$ denotes an arbitrary mass scale, introduced for dimensional reasons, which will eventually drop out in physical predictions.

Given an energy-momentum tensor $T_{\mu \nu}$, which in general contains vector and tensor contributions, the scalar parts $f_{v}$ and $f_{\pi}$ are determined by the identities:

$$
\begin{aligned}
i k^{j} T_{0 j}^{(s)} & =M^{2} k^{2} f_{v}, \\
-T_{i j}^{(s)} k^{i} k^{j}+\frac{1}{3} k^{2} \gamma^{k l} T_{k l}^{(s)} & =\frac{2}{3} M^{2} k^{4} f_{\pi} .
\end{aligned}
$$


On the other hand, $f_{v}$ and $f_{\pi}$ are related to $f_{\rho}$ and $f_{p}$, by the conservation equations $\nabla^{\nu} T_{\mu \nu}^{(s)}=0$ :

$$
\begin{gathered}
\dot{f}_{\rho}+k^{2} f_{v}+(\dot{a} / a)\left(f_{\rho}+3 f_{p}\right)=0, \\
\dot{f}_{v}+2(\dot{a} / a) f_{v}-f_{p}+(2 / 3) k^{2} f_{\pi}=0 .
\end{gathered}
$$

In the presence of seeds, and in the approximation in which perturbations are treated linearly, the total geometric perturbations can be separated into a part induced by the seeds, $\Psi_{s}, \Phi_{s}$, and a part induced by the perturbations of the cosmic fluid, $\Psi_{m}, \Phi_{m}$. The perturbed Einstein's equations (2.14) and (2.16) become

$$
\begin{aligned}
k^{2} \Phi & =4 \pi G \rho a^{2} D+\epsilon\left[f_{\rho}+3(\dot{a} / a) f_{v}\right], \\
\Phi+\Psi & =-8 \pi G a^{2} k^{-2} p \Pi-2 \epsilon f_{\pi},
\end{aligned}
$$

where $\epsilon \equiv 4 \pi G M^{2}$. If we define

$$
\Psi=\Psi_{s}+\Psi_{m}, \quad \Phi=\Phi_{s}+\Phi_{m}
$$

with:

$$
k^{2} \Phi_{s}=\epsilon\left[f_{\rho}+3(\dot{a} / a) f_{v}\right], \quad \Phi_{s}+\Psi_{s}=-2 \epsilon f_{\pi},
$$

we easily find

$$
\begin{aligned}
& \Phi_{m}=4 \pi G \rho a^{2} k^{-2}\left[D_{g}+3(1+w)\left(\frac{\dot{a}}{a}\right) \frac{V}{k}-3(1+w) \Phi\right], \\
& \Psi_{m}=-\Phi_{m}-8 \pi G a^{2} p \Pi k^{-2} .
\end{aligned}
$$

Equation (2.31) has been written in terms of the gaugeinvariant density perturbation $D_{g}$, because this choice will simplify our final equations. Physically, $D_{g}$ corresponds to the density perturbation in the flat slicing. The evolution of $D_{g}$ and $V$ is described by the conservation equations (2.18) and (2.19), which read explicitly:

$$
\begin{aligned}
\dot{D}_{g}+3\left(c_{s}^{2}-w\right) \frac{\dot{a}}{a} D_{g}= & -(1+w) k V, \\
\dot{V}+\frac{\dot{a}}{a}\left(1-3 c_{s}^{2}\right) V= & k\left(\Psi-3 c_{s}^{2} \Phi\right) \\
& +k \frac{c_{s}^{2}}{1+w} D_{g}-\frac{2 w}{3(1+w)} k \Pi .
\end{aligned}
$$

To simplify the analysis, we will assume $w=c_{s}^{2}$ $=$ constant. The unperturbed background equations are then solved by $a \propto \eta^{r}$, with $r=2 /(3 w+1)$. Since we are interested in very large scale perturbations in the cosmic microwave background, we concentrate our discussion on superhorizon scales, such that $k \eta \ll 1$. Equations (2.31) and (2.29) then lead to

$$
\Phi=\frac{1}{3(1+w)} D_{g}+\frac{r}{k \eta} V+\frac{2}{9 r^{2}(1+w)}(k \eta)^{2} \Phi_{s},
$$

where $r=1$ for the radiation-dominated era, and $r=2$ for the matter-dominated era.

The evolution equation for $D_{g}$, Eq. (2.33), implies $d D_{g} / d(k \eta)=-(1+w) V$. In the physical picture we have in mind, metric perturbations are triggered by the presence of the seeds alone, and we do not want to include an arbitrary contribution from the perturbations of the homogeneous sources. We thus require $D_{g}(0)=0$, which implies $D_{g}$ $\sim k \eta V$. Hence, we may neglect the $D_{g}$-term in Eq. (2.35) for $k \eta \ll 1$.

Combining Eqs. (2.35), (2.34), (2.28) we find, on superhorizon scales,

$$
\begin{aligned}
\Psi= & \frac{d V}{d(k \eta)}+\frac{r}{k \eta} V+\frac{2 w}{3 r^{2}(1+w)}(k \eta)^{2} \Phi_{s} \\
& +\frac{2(k \eta)^{2}}{9 r^{2}(1+w)}\left(2 \epsilon f_{\pi}+\Psi+\Phi\right) .
\end{aligned}
$$

The two equations (2.35) and (2.36) relate the three variables $\Psi, \Phi$ and $V$ once the seeds are given. To proceed, we need an equation of state to close the system. For single component fluids this equation usually takes the form $\Pi$ $=\Pi\left(D_{g}, V\right)$. We are interested in large-scale CMB anisotropies, which are induced at recombination and later, when the universe is already matter-dominated, with $p \ll \rho$. Thus, in what follows, we will consider the case $\Pi=0$, which implies

$$
\Phi+\Psi=-2 \epsilon f_{\pi} .
$$

Furthermore, in a matter-dominated Friedmann universe, $r$ $=2$ and $w=0$. The equation of motion for $V$, obtained by combining Eqs. (2.35), (2.36), (2.37), then reads

$$
\begin{aligned}
\frac{d V}{d(k \eta)}+\frac{4}{k \eta} V & =-\frac{1}{18}(k \eta)^{2} \Phi_{s}-2 \epsilon f_{\pi} \\
& =-\frac{1}{18} \eta^{2} \epsilon\left[f_{\rho}+3(\dot{a} / a) f_{v}\right]-2 \epsilon f_{\pi}
\end{aligned}
$$

In the next subsection, we shall see that the large-scale anisotropies of the CMB are determined by the combination $\Psi-\Phi$. Using Eqs. (2.35), (2.37) and (2.38), we find, immediately,

$$
\Psi-\Phi=\frac{d V}{d(k \eta)}-\frac{1}{18} \eta^{2} \epsilon\left[f_{\rho}+3(\dot{a} / a) f_{v}\right] .
$$

Modulo numbers of order unity, which can be computed case by case, we finally arrive at the estimates

$$
\Psi-\Phi \sim \frac{d V}{d(k \eta)} \sim \frac{V}{k \eta} \sim \max \left\{\epsilon f_{\pi}, \epsilon \eta^{2}\left(f_{\rho}+3 \frac{\dot{a}}{a} f_{v}\right)\right\} .
$$


Depending on whether $\eta^{2}\left(f_{\rho}+3(\dot{a} / a) f_{v}\right)$ or $f_{\pi}$ dominates in Eq. (2.40), we can distinguish between seeds with small and large anisotropic stresses. We will discuss in Sec. III to which case our string-cosmology seeds belong.

If the term $\epsilon \eta^{2}\left(f_{\rho}+3(\dot{a} / a) f_{v}\right)=x^{2} \Phi_{s}$ dominates, we conclude from Eqs. (2.38),(2.39) that

$$
\Phi \sim \Psi \sim(k \eta)^{2} \Phi_{s} \sim(k \eta)^{2} \Psi_{s} \ll \Phi_{s} \sim \Psi_{s},
$$

on super-horizon scales. This suppression of the total geometric perturbations, if compared with the source perturbations alone, is known under the name of "compensation" [14]. The conservation equations (2.18), (2.19) show that the presence of seeds induces matter perturbations that try to compensate the gravitational potential of the seeds. Since anisotropic stresses in the seeds cannot be compensated by a perfect fluid, compensation is not effective, if anisotropic stresses dominate. But, as shown here (see also [14]), the phenomenon of compensation is quite generic and, to a large extent, independent of the spectrum of seed fluctuations.

\section{B. The seed contribution to CMB anisotropies}

In this subsection we calculate the CMB anisotropies for models where perturbations are induced by seeds, and their contribution to $\Delta T / T$ via the Sachs-Wolfe effect [15]. We first discuss in general the motion of photons in a perturbed Friedmann universe.

We make use of the fact that the equations of motion of photons are conformally invariant. More precisely, two metrics that are conformally equivalent,

$$
d \bar{s}^{2}=a^{2} d s^{2},
$$

have the same light-like geodesics, only the corresponding affine parameters are different. Let us denote the two affine parameters by $\bar{\lambda}$ and $\lambda$ respectively, and the tangent vectors to the geodesic by

$$
n=\frac{d x}{d \lambda}, \quad \bar{n}=\frac{d x}{d \bar{\lambda}}, \quad n^{2}=\bar{n}^{2}=0, \quad n^{0}=1, \quad \mathbf{n}^{2}=1 .
$$

Setting $n^{0}=1+\delta n^{0}$, the geodesic equation for the perturbed metric

$$
d s^{2}=\left(\eta_{\mu \nu}+h_{\mu \nu}\right) d x^{\mu} d x^{\nu}
$$

yields, to first order,

$$
\left.\delta n^{0}\right|_{i} ^{f}=\left[h_{00}+h_{0 j} n^{j}\right]_{i}^{f}-\frac{1}{2} \int_{i}^{f} \dot{h}_{\mu \nu} n^{\mu} n^{\nu} d \lambda .
$$

On the other hand, the ratio of the energy of a photon measured by some observer at $t_{f}$ to the energy emitted at $t_{i}$ is

$$
\frac{E_{f}}{E_{i}}=\frac{(\bar{n} \cdot u)_{f}}{(\bar{n} \cdot u)_{i}}=\frac{T_{f}}{T_{i}} \frac{(n \cdot u)_{f}}{(n \cdot u)_{i}}
$$

where $u_{f}$ and $u_{i}$ are the four-velocities of the observer and emitter respectively, and the factor $T_{f} / T_{i}$ is the usual (unperturbed) redshift, which relates $n$ and $\bar{n}$. The velocity field of observer and emitter is given by

$$
u=(1-A) \partial_{\eta}+v^{i} \partial_{i} .
$$

An observer measuring a temperature $T_{0}$ receives photons that were emitted at the time $\eta_{d e c}$ of decoupling of matter and radiation, at the fixed temperature $T_{d e c}$. In first-order perturbation theory, we find the following relation between the unperturbed temperatures $T_{f}, T_{i}$, the measurable temperatures $T_{0}, T_{d e c}$, and the photon density perturbation:

$$
\frac{T_{f}}{T_{i}}=\frac{T_{0}}{T_{d e c}}\left(1-\frac{\delta T_{f}}{T_{f}}+\frac{\delta T_{i}}{T_{i}}\right)=\frac{T_{0}}{T_{d e c}}\left(1-\left.\frac{1}{4} \delta^{(\gamma)}\right|_{i} ^{f}\right),
$$

where $\delta^{(\gamma)}$ is the intrinsic density perturbation in the radiation and we used $\rho^{(\gamma)} \propto T^{4}$ in the last equality. Inserting the above equation and Eq. (2.45) into Eq. (2.46), and using Eq. (2.3) for the definition of $h_{\mu \nu}$, one finds, after integration by parts [18]:

$$
\begin{aligned}
\frac{E_{f}}{E_{i}}= & \frac{T_{0}}{T_{d e c}}\left\{1-\left[\frac{1}{4} D_{g}^{(\gamma)}+V_{j}^{(m)} n^{j}+\Psi-\Phi\right]_{i}^{f}\right. \\
& \left.+\int_{i}^{f}(\dot{\Psi}-\dot{\Phi}) d \lambda\right\} .
\end{aligned}
$$

Here $D_{g}^{(\gamma)}$ denotes the density perturbation in the radiation fluid, and $V^{(m)}$ is the peculiar velocity of the baryonic matter component (the emitter and observer of radiation). The final time values in the square bracket of Eq. (2.49) give rise only to monopole contributions and to the dipole due to our motion with respect to the $\mathrm{CMB}$, and will be neglected in what follows.

Evaluating Eq. (2.49) at final time $\eta_{0}$ (today) and initial time $\eta_{d e c}$, we obtain the temperature difference of photons coming from different directions $\mathbf{n}$ and $\mathbf{n}^{\prime}$

$$
\frac{\Delta T}{T} \equiv \frac{\delta T(\mathbf{n})}{T}-\frac{\delta T\left(\mathbf{n}^{\prime}\right)}{T},
$$

with temperature perturbation

$$
\begin{aligned}
\frac{\delta T(\mathbf{n})}{T}= & {\left[\frac{1}{4} D_{g}^{(\gamma)}+V_{j}^{(m)} n^{j}+\Psi-\Phi\right]\left(\eta_{d e c}, \mathbf{x}_{d e c}\right) } \\
& +\int_{\eta_{d e c}}^{\eta_{0}}(\dot{\Psi}-\dot{\Phi})(\eta, \mathbf{x}(\eta)) d \eta,
\end{aligned}
$$

where $\mathbf{x}(\eta)=\mathbf{x}_{0}-\left(\eta_{0}-\eta\right) \mathbf{n}$ is the unperturbed photon position at time $\eta$ for an observer at $\mathbf{x}_{0}$, and $\mathbf{x}_{d e c}=\mathbf{x}\left(\eta_{d e c}\right)$. The first term in Eq. (2.51) describes the intrinsic inhomogeneities on the surface of the last scattering, due to acoustic oscillations prior to decoupling. In general, it also contains contributions to the geometrical perturbations. This is especially important in the case of adiabatic inflationary models [23]. However, for perturbations induced by seeds, which 
satisfy the initial condition $D_{g}(k, \eta) \rightarrow 0$ for $\eta \rightarrow 0$, the geometrical contributions to $D_{g}$ can be neglected. The second term describes the relative motions of emitter and observer. This is the Doppler contribution to the CMB anisotropies. It appears on the same angular scales as the acoustic term, and we thus call the sum of the acoustic and Doppler contributions "acoustic peaks."

The last two terms are due to the inhomogeneities in the spacetime geometry; the first contribution determines the change in the photon energy due to the difference of the gravitational potential at the position of emitter and observer. Together with the part contained in $D_{g}^{(r)}$ they represent the "ordinary" Sachs-Wolfe effect. The second term accounts for red-shift or blue-shift caused by the time dependence of the gravitational field along the path of the photon, and represents the so-called Integrated Sachs-Wolfe (ISW) effect. The sum of the two terms is the full Sachs-Wolfe contribution (SW).

On angular scales $0.1^{\circ} \lesssim \theta \lesssim 2^{\circ}$, the main contribution to the CMB anisotropies comes from the acoustic peaks, while the SW effect is dominant on large angular scales. On scales smaller than about $0.1^{\circ}$, the anisotropies are damped by the finite thickness of the recombination shell, as well as by photon diffusion during recombination (Silk damping). Baryons and photons are very tightly coupled before recombination, and oscillate as a one-component fluid. During the process of decoupling, photons slowly diffuse out of over-dense regions into under-dense ones. To fully account for this process, one has to solve the Boltzmann equation for the photons (see, e.g. [18]).
The angular power spectrum of CMB anisotropies is expressed in terms of the dimensionless coefficients $C_{l}$, which appear in the expansion of the angular correlation function in terms of the Legendre polynomials $P_{l}$ :

$$
\begin{aligned}
& \left\langle\frac{\delta T}{T}(\mathbf{n}) \frac{\delta T}{T}\left(\mathbf{n}^{\prime}\right)\right\rangle_{\left(\mathbf{n} \cdot \mathbf{n}^{\prime}=\cos \vartheta\right)} \\
& \quad=\frac{1}{4 \pi} \sum_{l}(2 l+1) C_{l} P_{l}(\cos \vartheta) .
\end{aligned}
$$

Here the brackets denote spatial average, or expectation values if perturbations are quantized.

To determine the $C_{l}$ we Fourier-transform Eq. (2.51), defining

$$
\varphi(\mathbf{k})=\frac{1}{\sqrt{V}} \int_{V} \varphi(\mathbf{x}) e^{i \mathbf{k} \cdot \mathbf{x}} d^{3} x,
$$

and using the identity

$$
e^{i z \cos \vartheta}=\sum_{l}(2 l+1) i^{l} j_{l}(z) P_{l}(\cos \vartheta)
$$

(where $j_{l}$ is the spherical Bessel function of order $l$ ). For the coefficients $C_{l}$ of Eq. (2.52) we obtain

$$
C_{l}=\frac{2}{\pi} \int \frac{\left\langle\left|\Delta_{l}(\mathbf{k})\right|^{2}\right\rangle}{(2 l+1)^{2}} k^{2} d k
$$

where

$$
\begin{aligned}
\frac{\Delta_{l}}{2 l+1} & =j_{l}\left(k \eta_{0}\right)\left[\frac{1}{4} D_{g}^{(r)}\left(\mathbf{k}, \eta_{d e c}\right)+(\Psi-\Phi)\left(\mathbf{k}, \eta_{d e c}\right)\right]-j_{l}^{\prime}\left(k \eta_{0}\right) \mathbf{V}_{r}\left(\mathbf{k}, \eta_{d e c}\right)+\int_{\eta_{d e c}}^{\eta_{0}}(\dot{\Psi}-\dot{\Phi})\left(\mathbf{k}, \eta^{\prime}\right) j_{l}\left(k \eta_{0}-k \eta^{\prime}\right) d \eta^{\prime} \\
& =\frac{1}{4} D_{g}^{(r)}\left(\mathbf{k}, \eta_{d e c}\right) j_{l}\left(k \eta_{0}\right)-j_{l}^{\prime}\left(k \eta_{0}\right) \mathbf{V}_{r}\left(\mathbf{k}, \eta_{d e c}\right)+k \int_{\eta_{d e c}}^{\eta_{0}}(\Psi-\Phi)\left(\mathbf{k}, \eta^{\prime}\right) j_{l}^{\prime}\left(k \eta_{0}-k \eta^{\prime}\right) d \eta^{\prime}
\end{aligned}
$$

and $j_{l}^{\prime}$ stands for the derivative of $j_{l}$ with respect to its argument. On large angular scales, $k \eta_{d e c} \ll 1$ (which corresponds to $l \ll 100$ ), the SW contribution dominates:

$$
C_{l}^{S W}=\frac{2}{\pi} \int k^{4} d k\left\langle\left[\int_{\eta_{d e c}}^{\eta_{0}}(\Psi-\Phi)(\mathbf{k}, \eta) j_{l}^{\prime}\left(k \eta_{0}-k \eta\right) d \eta\right]^{2}\right\rangle .
$$

Let us approximate the Bardeen potentials on superhorizon scales by a power-law spectrum:

$$
\left\langle|\Psi-\Phi|^{2}\right\rangle=C^{2}(k)(k \eta)^{2 \gamma} .
$$

Furthermore, we consider models where the seed contribution does not grow in time on sub-horizon scales. In this case the Bardeen potentials, inside the horizon, are dominated by the cold dark matter contribution, which leads to timeindependent $\Phi$ and $\Psi$. We can thus approximate the Bardeen potentials by

$$
\Psi-\Phi \approx \begin{cases}C(k)(k \eta)^{\gamma}, & k \eta \ll 1, \\ C(k), & k \eta \gg 1 .\end{cases}
$$

We further assume that also $C(k)$ is given by a simple power law. Thus, for dimensional reasons, it has the form

$$
C(k)= \begin{cases}\mathcal{N} k^{-3 / 2}\left(k / k_{1}\right)^{\alpha}, & k \leqslant k_{1}, \\ 0, & k>k_{1},\end{cases}
$$


where $\mathcal{N}$ is a dimensionless constant, and $k_{1}$ denotes a comoving cutoff scale, i.e., the maximal amplified frequency determined by the explicit mechanism of seed production (in the case $\alpha=0$ no cutoff is needed). Inserting this in Eq. (2.57),

$$
C_{l}^{S W} \approx \mathcal{N}^{2} \frac{2}{\pi} \int_{0}^{k_{1}} \frac{d k}{k}\left(\frac{k}{k_{1}}\right)^{2 \alpha}|I(k)|^{2}
$$

where, setting $x=k \eta, x_{0}=k \eta_{0}, x_{d e c}=k \eta_{d e c}$,

$$
\begin{aligned}
I(k) & =\int_{x_{d e c}}^{1} d x x^{\gamma} j_{l}^{\prime}\left(x_{0}-x\right)+\int_{1}^{x_{0}} d x j_{l}^{\prime}\left(x_{0}-x\right) \\
& =\int_{x_{d e c}}^{1} d x x^{\gamma} j_{l}^{\prime}\left(x_{0}-x\right)+j_{l}\left(x_{0}-1\right) .
\end{aligned}
$$

We can see explicitly from this equation that the relevant contribution of each mode to the CMB anisotropy comes while the mode is still outside the horizon $(k \eta<1)$. We now distinguish two cases.

If $\gamma>-1$ the lower bound in Eq. (2.63) can be safely extended to 0 , and the integral is dominated by the region $k \eta \sim 1$, so that

$$
I(k) \sim j_{l}\left(x_{0}-x_{d e c}\right) \sim j_{l}\left(x_{0}\right), \quad x_{d e c} \ll 1<x_{0} .
$$

Inserting this in Eq. (2.61), the integral can be performed exactly (assuming $\eta_{0} k_{1} \gg l$ ), with the result, for $\alpha<1$,

$$
\begin{aligned}
C_{l}^{S W} \approx \mathcal{N}^{2}\left(k_{1} \eta_{0}\right)^{-2 \alpha} \frac{\Gamma(2-2 \alpha)}{4^{(1-\alpha)} \Gamma(3 / 2-\alpha)} \\
\times \frac{\Gamma(l+\alpha)}{\Gamma(l+2-\alpha)}, \quad \alpha<1
\end{aligned}
$$

(if $\alpha>1$, the integral grows towards large $k$ and is dominated by the contributions at $k \sim k_{1}$, leading to an l-independent result of order $\left.\left(\mathcal{N} / k_{1} \eta_{0}\right)^{2}\right)$. Comparing the above equation with the standard inflationary result [24],

$$
C_{l}^{S W} \propto \frac{\Gamma(l-1 / 2+n / 2)}{\Gamma(l+5 / 2-n / 2)},
$$

where $n$ denotes the usual spectral index, we find that $\alpha$ is related to $n$ by $\alpha=(n-1) / 2$. The scale-invariant spectrum, as it has been observed by the DMR experiment aboard the COBE satellite [25], requires

$$
0.9 \leqslant n \leqslant 1.5
$$

so that, within $1 \sigma$ error bars, the COBE observations imply

$$
-0.05 \leqslant \alpha \leqslant 0.25, \quad \gamma>-1 .
$$

Consider now the second case, $\gamma+1 \leqslant 0$. The integral (2.63) is now dominated by its value at the lower boundary and we get

$$
\begin{aligned}
|I(k)|^{2} \approx & \frac{1}{(\gamma+1)^{2}} x_{d e c}^{2(\gamma+1)}\left[\frac{l}{2 l+1} j_{l-1}\left(x_{0}\right)\right. \\
& \left.-\frac{l+1}{2 l+1} j_{l+1}\left(x_{0}\right)\right]^{2}
\end{aligned}
$$

If also $\alpha+\gamma<0$, the $k$-integral converges and we obtain (see Appendix A)

$$
\begin{aligned}
C_{l}^{S W} \approx & \frac{\mathcal{N}^{2}}{2^{2(\alpha+\gamma)}(\gamma+1)^{2}} \frac{\Gamma(-2(\alpha+\gamma))}{\Gamma(1 / 2-(\alpha+\gamma))^{2}}\left(\frac{\eta_{d e c}}{\eta_{0}}\right)^{2(\gamma+1)}\left(k_{1} \eta_{0}\right)^{-2 \alpha} \frac{\Gamma(l+1+\alpha+\gamma)}{\Gamma(l+1-\alpha-\gamma)} \frac{1}{(2 l+1)^{2}} \\
& \times\left[\frac{l^{2}(l-\alpha-\gamma)}{l+\alpha+\gamma}+\frac{2 l(l+1)(1 / 2+\alpha+\gamma)}{(1 / 2-\alpha-\gamma)}+\frac{(l+1)^{2}(l+1+\alpha+\gamma)}{(l+1-\alpha-\gamma)}\right] .
\end{aligned}
$$

Comparing again this last result with that of standard inflation, Eq. (2.66), and neglecting the weak $l$-dependence of $(2 l+1)^{-2}[\cdots]$ in Eq. (2.70), we obtain

$$
n \sim 3+2(\alpha+\gamma), \quad \alpha+\gamma<0 .
$$

(If, on the contrary, $\alpha+\gamma>0$, the coefficients $C_{l}$ are dominated by the large $k$ (i.e. small-scale) contribution, even for the very low values of $l$. In this case the small-scale perturbations become too large, which is excluded observationally by the fact that the spectrum, for CMB and matter perturbations, must be close to the Harrison-Zel'dovich spectrum [26].)
The observational limits on $n$ thus impose

$$
-0.05<\gamma+1+\alpha<0.25, \quad \gamma \leqslant-1, \quad n \simeq 3+2(\alpha+\gamma)
$$

and

$$
-0.05<\alpha<0.25,>-1, \quad n=1+2 \alpha .
$$

In the following sections we will apply these findings to electromagnetic and axionic seeds produced in string cosmology. In the axions case we will discuss separately massless and massive perturbations. 


\section{SEEDS FROM STRING COSMOLOGY}

In this section we compute the seed functions $f_{\rho}, f_{v}, f_{\pi}$, and we estimate the Bardeen potentials for electromagnetic and axion perturbations, including the case of massive axions.

\section{A. Amplification of quantum fluctuations}

We start by recalling the form of the (string-frame) lowenergy string effective action [27]:

$$
\begin{aligned}
\Gamma_{e f f}^{S}= & \int d^{D} x \sqrt{|g|} e^{-\phi}\left(R+g^{\mu \nu} \partial_{\mu} \phi \partial_{\nu} \phi\right. \\
& \left.-\frac{1}{12} g^{\mu \rho} g^{\nu \sigma} g^{\alpha \beta} H_{\mu \nu \alpha} H_{\rho \sigma \beta}-\frac{1}{4} g^{\mu \nu} g^{\rho \sigma} F_{\mu \rho} F_{\nu \sigma}\right),
\end{aligned}
$$

where we have included the antisymmetric tensor $H_{\mu \nu \alpha}$ $=\partial_{[\mu} B_{\nu \alpha]}$ and the $U(1)$ gauge field $F_{\mu \nu}=\partial_{[\mu} A_{\nu]}$. Note that this gauge field is typical of what emerges from heterotic string compactification. For gauge fields originating à la Kaluza-Klein, the action and the spectra are somewhat different, as discussed in [28].

Upon compactification down to four dimensions, and after introduction of the axion field $\sigma$ by the duality transformation:

$$
H^{\mu \nu \alpha}=e^{-\phi} \epsilon^{\mu \nu \alpha \beta} \partial_{\beta} \sigma,
$$

one easily arrives at the dimensionally reduced action:

$$
\begin{aligned}
\Gamma_{e f f}^{S}= & \int d^{4} x \sqrt{|g|} e^{-\phi}\left(R+g^{\mu \nu} \partial_{\mu} \phi \partial_{\nu} \phi\right. \\
& \left.-\frac{1}{2} e^{2 \phi} g^{\mu \nu} \partial_{\mu} \sigma \partial_{\nu} \sigma-\frac{1}{4} g^{\mu \nu} g^{\rho \sigma} F_{\mu \rho} F_{\nu \sigma}\right) .
\end{aligned}
$$

The study of tensor (T), scalar-dilaton (SD), electromagnetic (EM) and axion (AX) perturbations is conveniently performed defining the external " pump field,', responsible for their amplification. To this aim, we first identify for each perturbation the canonical variables $\psi^{i}$, which diagonalize the perturbed action expanded up to second order [29]. In a purely metric-dilaton background, such variables are easily found from Eq. (3.3) to be

$$
\begin{aligned}
\psi^{T} & =a e^{-\phi / 2} h^{T T} \equiv a_{E} h^{T T}, \quad \psi^{S D}=a e^{-\phi / 2} \phi+\cdots, \\
\psi^{E M} & =e^{-\phi / 2} A_{\mu}, \quad \psi^{A X}=a e^{\phi / 2} \sigma \equiv a_{A} \sigma .
\end{aligned}
$$

Here $h^{T T}$ denotes the transverse-traceless part of the metric perturbations, the dots in the equation for $\psi^{S D}$ represent the additional scalar-metric terms needed to reproduce the gauge-invariant scalar perturbation [29], $a_{E}$ is the scale factor in the Einstein frame, and $a_{A}$ in the axion frame [12]. By varying the perturbed action, we find that the Fourier modes $\psi_{k}(\eta)$ of each of these four perturbations satisfy decoupled, linear equations of the type:

$$
\ddot{\psi}_{k}+\left(k^{2}-\frac{\ddot{P}}{P}\right) \psi_{k}=0
$$

where $P(\eta)$ is the pump field, obtained for each case from Eq. (3.4) as

$$
P^{T}=P^{S D}=a_{E} ; \quad P^{E M}=e^{-\phi / 2} ; \quad P^{A X}=a_{A} .
$$

At the beginning of the inflationary era, characterized by an accelerated evolution of the pump field, every perturbation is well inside the horizon and Eq. (3.5) has oscillating solutions, which can be consistently normalized to a vacuum fluctuation spectrum. During the whole pre-big bang phase the general solution can be written in terms of Hankel functions [30], with a Bessel index determined by the power that characterizes the background evolution (in conformal time) of the pump field. This behavior has to be matched with the one after the pre-big-bang phase when, as we assume, the universe becomes radiation-dominated and the dilaton freezes to its present value. In all four cases this implies a free Klein-Gordon equation for the canonical variable after the period of accelerated evolution. By matching the pre-bigbang and radiation solutions of the perturbation equations, we eventually obtain the final amplified perturbations during the radiation era.

For $\mathrm{T}$ and SD perturbations the time evolution of the background leads to a spectrum that is in general too steep [7] (see also [31]) to be expected to give any significant contribution to very large scale structures, or to temperature anisotropies on the COBE scale. The only way to achieve a reasonable contribution would be to have a very long string phase with an almost constant dilaton [9], which is not excluded, in principle, either theoretically or phenomenologically, but which looks somewhat unlikely, from both points of view.

For EM perturbations, however, the situation seems to be more interesting. Consider in fact the transition from a prebig-bang phase, with growing dilaton $(\phi=-2 \beta \log |\eta|)$, to the standard radiation-dominated phase with $\phi=$ const, and call $\eta_{1}$ the transition time scale. The electromagnetic fluctuations are directly coupled to the dilaton background, in such a way that each polarization mode $\psi_{k}$ satisfies at all times, in momentum space and in the radiation gauge, the evolution equation:

$$
\ddot{\psi}_{k}+\left[k^{2}-e^{\phi / 2}\left(e^{-\phi / 2}\right)^{\bullet}\right] \psi_{k}=0 .
$$

In the pre-big-bang phase, the general solution of this equation, normalized to a vacuum fluctuation spectrum, can be written in terms of Hankel functions of the second kind as:

$$
\psi_{k}=\eta^{1 / 2} H_{\mu}^{(2)}(|k \eta|), \quad \mu=\left|\beta-\frac{1}{2}\right|, \quad \eta<\eta_{1} .
$$

In the radiation era we have instead the free plane-wave solution,

$$
\psi_{k}=\frac{1}{\sqrt{k}}\left[c_{+}(k) e^{-i k \eta}+c_{-}(k) e^{i k \eta}\right], \quad \eta>\eta_{1} .
$$


By matching the two solutions at the transition time $\eta_{1}$ we easily obtain, for $\left|k \eta_{1}\right| \ll 1$ and $\eta>\eta_{1}$,

$$
\begin{gathered}
c_{ \pm}= \pm c(k) e^{ \pm i k \eta}, \quad \psi_{k}=\frac{c(k)}{\sqrt{k}} \sin k\left|\eta-\eta_{1}\right|, \\
|c(k)| \simeq\left(k / k_{1}\right)^{-\mu-1 / 2},
\end{gathered}
$$

where $k_{1}=1 /\left|\eta_{1}\right|$ represents the maximal amplified frequency (higher-frequency modes are unaffected by the background transition). The associated energy-density distribution of the produced photons is then [11]:

$$
\begin{array}{r}
\frac{d \rho(k)}{d \log k} \simeq\left(\frac{k}{a}\right)^{4}\left|c_{-}(k)\right|^{2} \simeq\left(\frac{k_{1}}{a}\right)^{4}\left(\frac{k}{k_{1}}\right)^{3-2 \mu}, \\
k<k_{1}, \quad \mu<3 / 2,
\end{array}
$$

where $\mu<3 / 2$ to avoid photon overproduction which would destroy the homogeneity of the classical background, and where the amplitude $c(k)$ has been estimated modulo numerical factors of order 1 . At large times $\eta \gg\left|\eta_{1}\right|$ we thus obtain, in string cosmology, a cosmic background of electromagnetic fluctuations that, for a long enough pre-big-bang phase with $\beta \lesssim 2$, are characterized by a rather flat spectrum, and could provide the long-sought origin of the galactic magnetic fields [11]. The amplified fluctuations satisfy stochastic correlation functions, as a consequence of their quantum origin.

Correspondingly, if we consider axionic perturbations, we are led to the canonical equation

$$
\ddot{\psi}_{k}+\left(k^{2}-\frac{\ddot{a}_{A}}{a_{A}}\right) \psi_{k}=0,
$$

very similar to Eq. (3.7). The same procedure as in the electromagnetic case then leads to the spectrum (3.11) with $\mu$ $=|r|$, where $r$ parametrizes the three-dimensional axion scale factor as $a_{A}(\eta) \sim \eta^{r+1 / 2}$. For $r=-3 / 2$, in particular, the axion metric describes a de Sitter inflationary expansion, and the energy density of a massless axion background has a flat spectral distribution, $d \rho / d \log k \simeq\left(k_{1} / a\right)^{4}$, as first noted in [12]. The value of $r$ depends on the number and on the kinematics of the internal dimensions, and the value $-3 / 2$ can be obtained, in particular, for a ten-dimensional background with special symmetries [28].

In the axion case, however, the low frequency tail of the spectrum is further affected by the radiation $\rightarrow$ matter transition, as the axion pump field $a_{A}$ is not a constant (unlike the dilaton) in the matter-dominated era, where $a_{A}=a \propto \eta^{2}$. This has important consequences that will be discussed in detail in Sec. III C.

After these preliminary observations we shall now estimate the form of the seed functions for both EM and AX seeds.

\section{B. Electromagnetic seeds}

Here we determine the spectral components of the inhomogeneous stress tensor, for a stochastic background ob- tained by amplifying the quantum EM fluctuations of the vacuum, as discussed in the previous subsection. However, independently of the production mechanism, the results of this section can be applied to any EM fluctuation background parametrized by a vector potential that, in momentum space and in the radiation gauge, takes the form

$$
A_{i}(\mathbf{k}, \eta)=\frac{c_{i}(\mathbf{k})}{\sqrt{k}} \sin k \eta, \quad k_{i} A_{i}=0, \quad A_{0}=0 .
$$

$A_{i}$ is a Gaussian random variable which obeys the stochastic average condition:

$$
\begin{aligned}
\left\langle A_{i}(\mathbf{k}) A_{j}^{*}\left(\mathbf{k}^{\prime}\right)\right\rangle= & \frac{(2 \pi)^{3}}{2} \delta^{3}\left(k-k^{\prime}\right)\left(\delta_{i j}-\frac{k_{i} k_{j}}{k^{2}}\right) \\
& \times|\mathbf{A}(\mathbf{k}, \eta)|^{2} .
\end{aligned}
$$

The above condition has been normalized in such a way that

$$
\sum_{i}\left\langle A_{i}(\mathbf{k}) A_{i}^{*}\left(\mathbf{k}^{\prime}\right)\right\rangle=(2 \pi)^{3} \delta^{3}\left(k-k^{\prime}\right)|\mathbf{A}(\mathbf{k}, \eta)|^{2} .
$$

Taking into account that the electric component of the stochastic background is rapidly dissipated, because of the conductivity of the cosmic plasma [32], the seed stress tensor can be expressed in terms of the magnetic field only. Setting $B_{i}(k)=i \epsilon_{i j l} k_{j} A_{l}(k)$, the condition (3.14) implies

$$
\begin{aligned}
\left\langle B_{i}(\mathbf{k}) B_{j}^{*}\left(\mathbf{k}^{\prime}\right)\right\rangle= & \frac{(2 \pi)^{3}}{2} \delta^{3}\left(k-k^{\prime}\right)\left(\delta_{i j}-\frac{k_{i} k_{j}}{k^{2}}\right) \\
& \times|\mathbf{B}(\mathbf{k}, \eta)|^{2},
\end{aligned}
$$

where

$$
|\mathbf{B}(\mathbf{k}, \eta)|^{2}=k^{2}|\mathbf{A}(\mathbf{k}, \eta)|^{2}=k|\mathbf{c}(\mathbf{k})|^{2} \sin ^{2} k \eta .
$$

In a process of photon production, the coefficient $|\mathbf{c}(\mathbf{k})|^{2}$ represents the Bogoliubov coefficient [29] fixing the average photon number density, $\langle n(k)\rangle$, and is linked to the spectral energy distribution by

$$
\frac{d \rho(k)}{d \log k}=\left(\frac{k}{a}\right)^{4} \frac{\langle n(k)\rangle}{\pi^{2}} \simeq\left(\frac{k}{a}\right)^{4} \frac{|\mathbf{c}(\mathbf{k})|^{2}}{\pi^{2}} .
$$

In what follows we shall use for $|\mathbf{c}(\mathbf{k})|^{2}$ a power-law spectrum, characterized by a cut-off frequency $k_{1}$,

$$
|\mathbf{c}(\mathbf{k})|^{2}= \begin{cases}\left(k / k_{1}\right)^{-2 \mu-1}, & k \leqslant k_{1}, \quad \mu \leqslant 3 / 2, \\ 0, & k>k_{1} .\end{cases}
$$

This reproduces in particular the spectral distribution (3.11), where $\mu$ is fixed by the dilaton growth rate.

We shall now compute the two-point correlation functions, for the various components of the inhomogeneous stress tensor $T_{\mu}^{\nu}$, associated with the electromagnetic background: 


$$
\xi_{\mu}^{\nu}\left(x, x^{\prime}\right)=\left\langle T_{\mu}^{\nu}(x) T_{\mu}^{\nu}\left(x^{\prime}\right)\right\rangle-\left\langle T_{\mu}^{\nu}(x)\right\rangle\left\langle T_{\mu}^{\nu}\left(x^{\prime}\right)\right\rangle
$$

(no sum over $\mu, \nu$, and the angular brackets denote stochastic average). The Fourier transform of $\xi$ is related to the scalar seed functions $f_{\rho}, f_{v}, f_{\pi}$, defined in the previous section. For $\xi_{0}^{0}$ we have, for instance,

$$
\xi_{0}^{0}\left(x, x^{\prime}\right)=\left(\frac{M}{a}\right)^{4} \int \frac{d^{3} k}{(2 \pi)^{3}} e^{i \mathbf{k} \cdot\left(\mathbf{x}-\mathbf{x}^{\prime}\right)}\left|f_{\rho}(k)\right|^{2} .
$$

For $E_{i}=0$, in particular, we have to compute the correlation of a sum of terms that are quadratic in the magnetic field. We start considering the energy-density correlation function,

$$
\xi_{0}^{0}\left(x, x^{\prime}\right)=\left\langle\rho(x) \rho\left(x^{\prime}\right)\right\rangle-(\langle\rho(x)\rangle)^{2}, \quad \rho=-T_{0}^{0}=\frac{|\mathbf{B}|^{2}}{8 \pi a^{4}},
$$

and compute

$$
\Delta_{i j}^{B}\left(x, x^{\prime}\right)=\left\langle B_{i}^{2}(x) B_{j}^{2}\left(x^{\prime}\right)\right\rangle-\left\langle B_{i}^{2}\right\rangle\left\langle B_{j}^{2}\right\rangle
$$

where, using the stochastic average (3.16) and the reality condition $B^{*}(k)=B(-k)$,

$$
\left\langle B_{i}^{2}(x)\right\rangle=\frac{1}{2} \int \frac{d^{3} k}{(2 \pi)^{3}}|\mathbf{B}(k)|^{2}\left(1-\frac{k_{i}^{2}}{k^{2}}\right) .
$$

In momentum space, the two-point correlation function for the energy density can be written as a four-point correlation function for the stochastic fields (see also [33]). We have, in particular,

$$
\begin{aligned}
& \left\langle B_{i}^{2}(x) B_{j}^{2}\left(x^{\prime}\right)\right\rangle \\
& =\int \frac{d^{3} k}{(2 \pi)^{3}} \frac{d^{3} k^{\prime}}{(2 \pi)^{3}} \frac{d^{3} p}{(2 \pi)^{3}} \frac{d^{3} q}{(2 \pi)^{3}} e^{i\left(\mathbf{k} \cdot \mathbf{x}+\mathbf{k}^{\prime} \cdot \mathbf{x}^{\prime}\right)} \\
& \quad \times\left\langle B_{i}(p) B_{i}(k-p) B_{j}(q) B_{j}\left(k^{\prime}-q\right)\right\rangle
\end{aligned}
$$

Decomposing the four-point bracket of the Gaussian variables $B_{j}$ as

$$
\begin{aligned}
& \left\langle B_{i}(p) B_{i}(k-p)\right\rangle\left\langle B_{j}(q) B_{j}\left(k^{\prime}-q\right)\right\rangle \\
& +\left\langle B_{i}(p) B_{j}(q)\right\rangle\left\langle B_{i}(k-p) B_{j}\left(k^{\prime}-q\right)\right\rangle \\
& \quad+\left\langle B_{i}(p) B_{j}\left(k^{\prime}-q\right)\right\rangle\left\langle B_{i}(k-p) B_{j}(q)\right\rangle,
\end{aligned}
$$

and using Eq. (3.16), we find that the first term in the above equation is exactly cancelled by the quadratic averages $\left\langle B_{i}^{2}\right\rangle\left\langle B_{j}^{2}\right\rangle$, while the other two terms give (no sum over $i, j$ ):

$$
\begin{aligned}
\Delta_{i j}^{B}\left(x, x^{\prime}\right)= & \frac{1}{2} \int \frac{d^{3} k}{(2 \pi)^{3}} \frac{d^{3} p}{(2 \pi)^{3}} e^{i \mathbf{k} \cdot \Delta \mathbf{x}}|\mathbf{B}(\mathbf{p})|^{2} \\
& \times|\mathbf{B}(\mathbf{k}-\mathbf{p})|^{2}\left(\delta_{i j}-\frac{p_{i} p_{j}}{p^{2}}\right) \\
& \times\left(\delta_{i j}-\frac{(k-p)_{i}(k-p)_{j}}{|\mathbf{k}-\mathbf{p}|^{2}}\right),
\end{aligned}
$$

where $\Delta x=x-x^{\prime}$. By summing over the vector components we obtain

$$
\begin{aligned}
\Delta^{B}\left(x, x^{\prime}\right)= & \sum_{i j} \Delta_{i j}^{B}\left(x, x^{\prime}\right) \\
= & \frac{1}{2} \int \frac{d^{3} k}{(2 \pi)^{3}} \frac{d^{3} p}{(2 \pi)^{3}} e^{i \mathbf{k} \cdot \Delta \mathbf{x}}|\mathbf{B}(\mathbf{p})|^{2}|\mathbf{B}(\mathbf{k}-\mathbf{p})|^{2} \\
& \times\left[1+\frac{|\mathbf{p} \cdot(\mathbf{k}-\mathbf{p})|^{2}}{p^{2}|\mathbf{k}-\mathbf{p}|^{2}}\right]
\end{aligned}
$$

According to Eq. (3.21), the energy-density spectrum of the electromagnetic seeds is thus determined by

$$
\begin{aligned}
\left|f_{\rho}\right|^{2}\left(\frac{M}{a}\right)^{4}= & \frac{1}{2\left(8 \pi a^{4}\right)^{2}} \int \frac{d^{3} p}{(2 \pi)^{3}} \\
& \times|\mathbf{c}(\mathbf{p})|^{2}|\mathbf{c}(\mathbf{k}-\mathbf{p})|^{2} p|\mathbf{k}-\mathbf{p}| \\
& \times\left(1+\cos ^{2} \alpha\right) \sin ^{2} p \eta \sin ^{2}|\mathbf{k}-\mathbf{p}| \eta
\end{aligned}
$$

where $\alpha$ is the angle between $\mathbf{p}$ and $\mathbf{k}-\mathbf{p}$. Inserting the power spectrum (3.19), and defining $\mathbf{y}=\mathbf{p} / k_{1}, \mathbf{z}=\mathbf{k} / k_{1}$, the above integral can be written, in polar coordinates, as

$$
\begin{aligned}
\left|f_{\rho}\right|^{2}\left(\frac{M}{a}\right)^{4}= & \frac{k_{1}^{5}}{2\left(8 \pi a^{4}\right)^{2}(2 \pi)^{2}} \int_{0}^{1} d y y^{2-2 \mu} \\
& \times \int_{-1}^{1} d x \beta^{-2 \mu}\left(1+\cos ^{2} \alpha\right) \\
& \times \sin ^{2}\left(y k_{1} \eta\right) \sin ^{2}\left(\beta k_{1} \eta\right),
\end{aligned}
$$

where we defined $x=\cos \vartheta, \vartheta$ being the angle between $\mathbf{p}$ and $\mathbf{k}$, and

$$
\begin{aligned}
\beta^{2}=|\mathbf{z}-\mathbf{y}|^{2} & =y^{2}+z^{2}-2 x y z, \\
\cos ^{2} \alpha & =\beta^{-2}\left(y^{2}+x^{2} z^{2}-2 x y z\right) .
\end{aligned}
$$

The integral of Eq. (3.30) will be evaluated for $|k \eta|$ $=\left|z k_{1} \eta\right| \ll 1$, since we are interested in the large-scale sector 
of the CMB anisotropy, namely in the spectrum of all modes that are still outside the horizon at the time of decoupling. For EM seeds these modes give the dominant contribution to the SW effect, as we will see in Sec. IV. Estimating the contributions to the integral from the regions $p \eta \ll 1, p \eta$ $\sim 1$ and $p \eta \gg 1$, and recalling that $\mu \leqslant 3 / 2$ according to Eq.
(3.11), we find that the dominant contribution comes from $p \eta \gg 1$ if $\mu \leqslant 3 / 4$. If $3 / 4 \leqslant \mu \leqslant 3 / 2$, the integral is dominated from its contribution at $p \sim k$, thus $p \eta<1$ on super-horizon scales. In both cases we obtain for $f_{\rho}$ a white noise spectrum, i.e. $\left|f_{\rho}(k)\right|^{2} \sim$ constant, but in the second case there is a parametric enhancement (see Appendix B). More precisely

$$
k^{3}\left|f_{\rho}\right|^{2}\left(\frac{M}{a}\right)^{4} \simeq \begin{cases}d_{\rho}^{2}\left(k_{1} / a\right)^{8}\left(k / k_{1}\right)^{3}, & \mu \leqslant 3 / 4 \\ c_{\rho}^{2}\left(k_{1} / a\right)^{8}\left(k / k_{1}\right)^{3}\left(k_{1} \eta\right)^{4 \mu-3}, & 3 / 4 \leqslant \mu \leqslant 3 / 2,\end{cases}
$$

where $d_{\rho}$ and $c_{\rho}$ are dimensionless numbers of order 1 . Consequently, the energy-density contribution of the EM seeds to the Bardeen potentials is, according to Eq. (2.40),

$$
\epsilon \eta^{2}\left|f_{\rho}\right| k^{3 / 2} \simeq \begin{cases}4 \pi G d_{\rho}(a \eta)^{2}\left(k_{1} / a\right)^{4}\left(k / k_{1}\right)^{3 / 2}, & \mu \leqslant 3 / 4, \\ 4 \pi G c_{\rho}(a \eta)^{2}\left(k_{1} / a\right)^{4}\left(k / k_{1}\right)^{3 / 2}\left(k_{1} \eta\right)^{2 \mu-3 / 2}, & 3 / 4 \leqslant \mu \leqslant 3 / 2 .\end{cases}
$$

The contribution of the off-diagonal scalar potential $f_{\pi}$ can be similarly obtained by computing the correlation function $\xi_{i}^{j}\left(x, x^{\prime}\right)$, with $i \neq j$. For purely magnetic seeds, $f_{v}=0$, we find

$$
f_{\rho}=3 f_{p} \sim k^{2} f_{\pi}
$$

so that the Bardeen potentials, according to Eq. (2.40), are always dominated by $f_{\pi}$ on super-horizon scales, as $\eta^{2} f_{\rho} / f_{\pi}$ $\sim(k \eta)^{2} \ll 1$. Therefore

$$
k^{3 / 2}|\Psi-\Phi| \sim \epsilon k^{3 / 2}\left|f_{\pi}\right| \simeq \begin{cases}4 \pi G d_{\pi}(a \eta)^{2}\left(k_{1} / a\right)^{4}\left(k / k_{1}\right)^{-1 / 2}\left(k_{1} \eta\right)^{-2}, & \mu \leqslant 3 / 4, \\ 4 \pi G c_{\pi}(a \eta)^{2}\left(k_{1} / a\right)^{4}\left(k / k_{1}\right)^{-1 / 2}\left(k_{1} \eta\right)^{2 \mu-7 / 2}, & 3 / 4 \leqslant \mu \leqslant 3 / 2 .\end{cases}
$$

where $d_{\pi}$ and $c_{\pi}$ are dimensionless numbers of order 1 . By assuming that the universe becomes immediately radiationdominated at the physical cut-off scale $H_{1}=k_{1} / a_{1}$, such a fluctuation spectrum can be expressed in terms of $\Omega \gamma(\eta)$ $=\left(H_{1} / H\right)^{2}\left(a_{1} / a\right)^{4}$, i.e. of the fraction of critical energy density in radiation at a given time $\eta$, and of $g_{1}=H_{1} / M_{p}$, the transition scale in units of the Planck mass $M_{p}$. Denoting with $\omega=k / a$ the proper frequency, and using $\rho_{c}=3 M_{p}^{2} H^{2} / 8 \pi$ for the critical density, we obtain

$$
k^{3 / 2}|\Psi-\Phi| \sim \begin{cases}g_{1}^{2} \Omega_{\gamma}(\eta)\left(\omega / \omega_{1}\right)^{-1 / 2}\left(\omega_{1} / H\right)^{-2}, & \mu \leqslant 3 / 4, \\ g_{1}^{2} \Omega_{\gamma}(\eta)\left(\omega / \omega_{1}\right)^{-1 / 2}\left(\omega_{1} / H\right)^{2 \mu-7 / 2}, & 3 / 4 \leqslant \mu \leqslant 3 / 2 .\end{cases}
$$

\section{Axionic seeds}

As a second example of seed inhomogeneities we will consider a pseudoscalar stochastic background, amplified according to the perturbation equation (3.12).

In the initial, higher-dimensional pre-big-bang phase, i.e. for $\eta<\eta_{1}$, the solution for the canonical variable $\psi$ can be written as in Eq. (3.8), with $\mu=|r| \leqslant 3 / 2$, as discussed previously. In the radiation era, i.e. for $\eta_{1}<\eta<\eta_{e q}$, the effective potential $\ddot{a}_{A} / a_{A}$ is vanishing, as $\phi=$ const and $a \sim \eta$, and $\psi$ is given by the plane-wave solution (3.9). In the final matter-dominated era, i.e. for $\eta>\eta_{e q}$, we have $a \sim \eta^{2}$, and $\ddot{a}_{A} / a_{A}=2 / \eta^{2}$. The plane-wave solution is still valid for modes with $k>k_{e q}=\eta_{e q}^{-1}$, that are unaffected by the last transitions. Modes with $k<k_{e q}$ feel instead the effect of the potential in the matter era, and the general solution of Eq. (3.12), for those modes, can be written as

$$
\begin{aligned}
\psi_{k}(\eta)= & \frac{\sqrt{k \eta}}{\sqrt{k}}\left(A H_{3 / 2}^{(2)}+B H_{3 / 2}^{(1)}\right) \\
= & \frac{\sqrt{k \eta}}{\sqrt{k}}\left[(A+B) J_{3 / 2}-i(A-B) Y_{3 / 2}\right], \\
& k<k_{e q}, \quad \eta>\eta_{e q} .
\end{aligned}
$$

Here $J_{3 / 2}$ and $Y_{3 / 2}$ are Bessel functions of argument $k \eta$ (we follow the conventions of [30]).

The matching of the solutions at $\eta_{1}$ determines the coefficients $c_{ \pm}(k)$ as in Eq. (3.10). The matching at $\eta_{e q}$ gives

$$
A+B \sim c(\mathbf{k})\left(k \eta_{e q}\right)^{-1}, \quad A-B \sim c(\mathbf{k})\left(k \eta_{e q}\right)^{2},
$$


so that the contribution of $J_{3 / 2}$ to $\psi_{k}$ is always dominant with respect to the $Y_{3 / 2}$ contribution, both for $k \eta>1$ and $k \eta<1$. In the matter-dominated era, i.e. for $\eta>\eta_{e q}$, we can thus approximate the produced stochastic axion background as follows:

$$
\begin{aligned}
\sigma(\mathbf{k}, \eta) & \simeq \frac{c(\mathbf{k})}{a \sqrt{k}} \sin k \eta, \quad k>k_{e q}, \\
& \simeq \frac{c(\mathbf{k})}{a \sqrt{k}}\left(\frac{k}{k_{e q}}\right)^{-1}(k \eta)^{2}, \quad k<k_{e q}, \quad k \eta<1, \\
& \simeq \frac{c(\mathbf{k})}{a \sqrt{k}}\left(\frac{k}{k_{e q}}\right)^{-1}, \quad k<k_{e q}, \quad k \eta>1 .
\end{aligned}
$$

The correlation functions for the various components of the stress tensor,

$$
T_{\mu}^{\nu}=\partial_{\mu} \sigma \partial^{\nu} \sigma-\frac{1}{2} \delta_{\mu}^{\nu}\left(\partial_{\alpha} \sigma\right)^{2}
$$

can be computed by exploiting the stochastic average conditions of the Gaussian variables $\sigma, \dot{\sigma}$ and $\sigma_{j}=\partial_{j} \sigma$,

$$
\begin{aligned}
\left\langle\sigma(\mathbf{k}) \sigma^{*}\left(\mathbf{k}^{\prime}\right)\right\rangle & =(2 \pi)^{3} \delta^{3}\left(k-k^{\prime}\right) \Sigma_{1}(\mathbf{k}, \eta), \\
\left\langle\dot{\sigma}(\mathbf{k}) \dot{\sigma}^{*}\left(\mathbf{k}^{\prime}\right)\right\rangle & =(2 \pi)^{3} \delta^{3}\left(k-k^{\prime}\right) \Sigma_{2}(\mathbf{k}, \eta), \\
\left\langle\sigma_{i}(\mathbf{k}) \sigma_{j}^{*}\left(\mathbf{k}^{\prime}\right)\right\rangle & =k_{i} k_{j}(2 \pi)^{3} \delta^{3}\left(k-k^{\prime}\right) \Sigma_{1}(\mathbf{k}, \eta), \\
\left\langle\sigma_{j}(\mathbf{k}) \dot{\sigma}^{*}\left(\mathbf{k}^{\prime}\right)\right\rangle & =-\left\langle\dot{\sigma}(\mathbf{k}) \sigma_{j}^{*}\left(\mathbf{k}^{\prime}\right)\right\rangle \\
& =i k_{j}(2 \pi)^{3} \delta^{3}\left(k-k^{\prime}\right) \Sigma_{3}(\mathbf{k}, \eta),
\end{aligned}
$$

where, according to Eq. (3.39),

$$
\begin{aligned}
\Sigma_{1}(\mathbf{k}, \eta) & \simeq \frac{|c(\mathbf{k})|^{2}}{k a^{2}}, \quad k>k_{e q}, \\
& \simeq \frac{|c(\mathbf{k})|^{2}}{k a^{2}}\left(\frac{k}{k_{e q}}\right)^{-2}(k \eta)^{4}, \quad k<k_{e q}, \quad k \eta<1, \\
& \simeq \frac{|c(\mathbf{k})|^{2}}{k a^{2}}\left(\frac{k}{k_{e q}}\right)^{-2}, \quad k<k_{e q}, \quad k \eta>1
\end{aligned}
$$

$$
\begin{aligned}
\Sigma_{2}(\mathbf{k}, \eta) & \simeq k \frac{|c(\mathbf{k})|^{2}}{a^{2}}, \quad k>k_{e q}, \\
& \simeq 0, \quad k<k_{e q}, \quad k \eta<1, \\
& \simeq k \frac{|c(\mathbf{k})|^{2}}{a^{2}}\left(\frac{k}{k_{e q}}\right)^{-2}, \quad k<k_{e q}, \quad k \eta>1 \\
\Sigma_{3}(\mathbf{k}, \eta) & \simeq \frac{|c(\mathbf{k})|^{2}}{a^{2}}, \quad k>k_{e q}, \\
& \simeq 0, \quad k<k_{e q}, \quad k \eta<1, \\
& \simeq \frac{|c(\mathbf{k})|^{2}}{a^{2}}\left(\frac{k}{k_{e q}}\right)^{-2}, \quad k<k_{e q}, \quad k \eta>1
\end{aligned}
$$

Following the same procedure as the one used for EM seeds, and collecting all contributions to the correlation function of the axion energy density,

$$
\rho_{\sigma}=\frac{1}{2 a^{2}}\left[\dot{\sigma}^{2}+\left(\partial_{i} \sigma\right)^{2}\right]
$$

we obtain from $\xi_{0}^{0}\left(x, x^{\prime}\right)$ that the energy density spectrum is determined by

$$
\begin{aligned}
k^{3}\left|f_{\rho}\right|^{2}\left(\frac{M}{a}\right)^{4}= & \frac{2 k^{3}}{\left(2 a^{2}\right)^{2}} \int \frac{d^{3} p}{(2 \pi)^{3}}\left[\Sigma_{2}(\mathbf{p}) \Sigma_{2}(\mathbf{k}-\mathbf{p})\right. \\
& +|\mathbf{p} \cdot(\mathbf{k}-\mathbf{p})|^{2} \Sigma_{1}(\mathbf{p}) \Sigma_{1}(\mathbf{k}-\mathbf{p}) \\
& \left.-2 \mathbf{p} \cdot(\mathbf{k}-\mathbf{p}) \Sigma_{3}(\mathbf{p}) \Sigma_{3}(\mathbf{k}-\mathbf{p})\right]
\end{aligned}
$$

In order to evaluate this integral outside the horizon, in the region $k \eta \leqslant 1$, we must distinguish two cases, $\mu<3 / 4$ and $\mu>3 / 4$. In both cases, by separate integration in the ranges $0<p<\eta^{-1}, \eta^{-1}<p<k_{e q}, k_{e q}<p<k_{1}$, we find a white noise spectrum, $\left|f_{\rho}\right| \sim$ const. In particular (see Appen$\operatorname{dix} \mathrm{B})$,

$$
k^{3 / 2}\left|f_{\rho}\right|\left(\frac{M}{a}\right)^{2}= \begin{cases}d_{\rho}^{\sigma}\left(k_{1} / a\right)^{4}\left(k / k_{1}\right)^{3 / 2}\left[1+\delta_{\rho}^{\sigma}\left(k_{e q} / k_{1}\right)^{2}\left(k_{1} \eta\right)^{2 \mu+1 / 2}\right], & \mu \leqslant 3 / 4 \\ c_{\rho}^{\sigma}\left(k_{1} / a\right)^{4}\left(k / k_{1}\right)^{3 / 2}\left(k_{e q} / k_{1}\right)^{2}\left(k_{1} \eta\right)^{2 \mu+1 / 2}, & 3 / 4 \leqslant \mu \leqslant 3 / 2,\end{cases}
$$

where $c_{\rho}^{\sigma}, d_{\rho}^{\sigma}, \delta_{\rho}^{\sigma}$ are dimensionless numbers of order 1 . The same power spectrum is also obtained for the scalar velocity potential $f_{v}$, associated to the axion seeds. An explicit computation gives in fact $k f_{v} \sim k \eta f_{\rho}$ so that the contribution of $f_{\rho}$ and $f_{v}$ to the Bardeen potential are both of the same order: namely,

$$
\epsilon \eta^{2}\left|f_{\rho}\right| k^{3 / 2} \sim \epsilon \eta^{2} \frac{\dot{a}}{a}\left|f_{v}\right| k^{3 / 2}= \begin{cases}4 \pi G d_{\rho}^{\sigma}(a \eta)^{2}\left(k_{1} / a\right)^{4}\left(k / k_{1}\right)^{3 / 2}\left[1+\delta_{\rho}^{\sigma}\left(k_{e q} / k_{1}\right)^{2}\left(k_{1} \eta\right)^{2 \mu+1 / 2}\right], & \mu \leqslant 3 / 4 \\ 4 \pi G c_{\rho}^{\sigma}(a \eta)^{2}\left(k_{1} / a\right)^{4}\left(k / k_{1}\right)^{3 / 2}\left(k_{e q} / k_{1}\right)^{2}\left(k_{1} \eta\right)^{2 \mu+1 / 2,} & 3 / 4 \leqslant \mu \leqslant 3 / 2 .\end{cases}
$$


We will now consider the anisotropic stress potential $f_{\pi}$, defined according to Eq. (2.23) by

$$
\nabla^{4} f_{\pi}=\frac{3}{2 M^{2}} \partial_{i} \partial_{j}\left[\sigma_{i} \sigma_{j}-\frac{1}{3} \delta_{i j}\left(\partial_{k} \sigma\right)^{2}\right], \quad \nabla^{2}=\delta_{i j} \partial_{i} \partial_{j}
$$

Summing all contributions in the two point correlation function, we find

$$
\begin{aligned}
& \left\langle\nabla^{4} f_{\pi}(x) \nabla^{4} f_{\pi}\left(x^{\prime}\right)\right\rangle-\left(\left\langle\nabla^{4} f_{\pi}\right\rangle\right)^{2}=\frac{9}{2 M^{4}} \int \frac{d^{3} k}{(2 \pi)^{3}} e^{i \mathbf{k} \cdot \Delta \mathbf{x}_{k}} \int \frac{d^{3} p}{(2 \pi)^{3}} p^{2}|\mathbf{k}-\mathbf{p}|^{2} \Sigma_{1}(\mathbf{p}) \Sigma_{1}(\mathbf{k}-\mathbf{p}) \\
& \times\left(\cos ^{2} \vartheta \cos ^{2} \gamma-\frac{1}{3} \cos \vartheta \cos \gamma \cos \alpha+\frac{1}{9} \cos ^{2} \alpha\right),
\end{aligned}
$$

where $\vartheta, \alpha$ and $\gamma$ are, respectively, the angles between $\mathbf{p}$ and $\mathbf{k}, \mathbf{p}$ and $\mathbf{k}-\mathbf{p}$ and $\mathbf{k}$ and $\mathbf{k}-\mathbf{p}$. The integral over $p$ is of the same type as the integral for the energy density spectrum [see Eq. (3.46)], and gives for $k^{2} f_{\pi}$ the same white noise spectrum (3.47) as for $f_{\rho}$ (modulo numbers of order 1$)$, since

$$
k^{3 / 2}\left|f_{\pi}(k)\right|\left(\frac{k}{a}\right)^{2} M^{2} \sim k^{3 / 2}\left|f_{\rho}(k)\right|\left(\frac{M}{a}\right)^{2} .
$$

On super-horizon scales the contribution of $f_{\pi}$ to the Bardeen potentials is always dominant with respect to the $f_{\rho}$ contribution since, from the above equation,

$$
\eta^{2} f_{\rho} \sim(k \eta)^{2} f_{\pi}
$$

In the whole range $k \eta \leqslant 1$ we can thus estimate the scalar perturbation spectrum, induced by massless axion seeds, through the $f_{\pi}$ contribution to the Bardeen potentials. We find

$$
\begin{aligned}
k^{3 / 2}|\Psi-\Phi| \sim \epsilon k^{3 / 2}\left|f_{\pi}\right| & = \begin{cases}4 \pi G d_{\pi}^{\sigma}(a \eta)^{2}\left(k_{1} / a\right)^{4}\left(k / k_{1}\right)^{-1 / 2}\left(k_{1} \eta\right)^{-2}\left[1+\delta_{\rho}^{\sigma}\left(k_{e q} / k_{1}\right)^{2}\left(k_{1} \eta\right)^{2 \mu+1 / 2}\right], & \mu \leqslant 3 / 4, \\
4 \pi G c_{\pi}^{\sigma}(a \eta)^{2}\left(k_{1} / a\right)^{4}\left(k / k_{1}\right)^{-1 / 2}\left(k_{e q} / k_{1}\right)^{2}\left(k_{1} \eta\right)^{2 \mu-3 / 2}, & 3 / 4 \leqslant \mu \leqslant 3 / 2,\end{cases} \\
& \sim \begin{cases}g_{1}^{2} \Omega_{\gamma}(\eta)\left(\omega / \omega_{1}\right)^{-1 / 2}\left(\omega_{1} / H\right)^{-2}\left[1+\delta_{\rho}^{\sigma}\left(\omega_{e q} / \omega_{1}\right)^{2}\left(\omega_{1} / H\right)^{2 \mu+1 / 2}\right], & \mu \leqslant 3 / 4, \\
g_{1}^{2} \Omega_{\gamma}(\eta)\left(\omega / \omega_{1}\right)^{-1 / 2}\left(\omega_{e q} / \omega_{1}\right)^{2}\left(\omega_{1} / H\right)^{2 \mu-3 / 2}, & 3 / 4 \leqslant \mu \leqslant 3 / 2,\end{cases}
\end{aligned}
$$

where $c_{\pi}^{\sigma}, d_{\pi}^{\sigma}, \delta_{\pi}^{\sigma}$ are dimensionless numbers of order 1. As we will see in Sec. IV, the dominant contribution to the SW effect now comes, for each mode, from the time of reentry $\eta \sim 1 / k$.

Let us finally discuss the case of massive axions, with

$$
T_{\mu}^{\nu}=\partial_{\mu} \sigma \partial^{\nu} \sigma-\frac{1}{2} \delta_{\mu}^{\nu}\left[\left(\partial_{\alpha} \sigma\right)^{2}-m^{2} \sigma^{2}\right]
$$

and a primordial distribution again characterized by the in$\operatorname{dex} \mu$, as in Eq. (3.19). The mass term directly contribute to $f_{\rho}$ and $f_{p}$, and only indirectly to the off-diagonal potentials $f_{v}, f_{\pi}$. We are interested in the axion perturbations that may be relevant to the large-scale CMB anisotropy, namely in the modes that are outside the horizon at the decoupling era, $k$ $<a H_{d e c}$. If, for these modes, the mass contribution is negligible, $m a<k<a H_{d e c}$, then the $\mathrm{AX}$ seed functions and the corresponding Bardeen potentials are the same as in the massless case (see before). We will thus concentrate our discussion on the case in which the axion mass is large enough, so that all modes outside the horizon at the equilibrium epoch are already non-relativistic:

$$
m a>a H_{e q}>k \text {. }
$$

In this case we may neglect the effects of an additional axion production in the matter-dominated era, since $a^{2} m^{2}>\ddot{a} / a$ at $\eta \geqslant \eta_{e q}$. The axion fluctuations are amplified by the inflation $\rightarrow$ radiation transition, but are to be evaluated in the non-relativistic regime $\left(\eta>\eta_{e q}\right)$, where the mass contribution is already important.

For non-relativistic, super-horizon modes, the Fourier components of the axion field become (see the non-trivial calculation reported in Appendix C):

$$
\begin{gathered}
\sigma(\mathbf{k}, \eta)=\frac{c(\mathbf{k})}{a \sqrt{m a}}\left(\frac{k}{k_{1}}\right)^{1 / 2}\left(\frac{H_{1}}{m}\right)^{1 / 4} \sin \left(\frac{m}{H}\right), \\
k<k_{m}=k_{1}\left(\frac{m}{H_{1}}\right)^{1 / 2},
\end{gathered}
$$

where the initial distribution $c(k)$ is still given by Eq. (3.19). Here $k_{m}$ is the limiting frequency re-entering the horizon at the same time as it becomes non-relativistic, i.e. $k_{m} / a_{m}$ $=H_{m}=m$. Indeed, we are assuming that at the transition scale $H_{1}$ the mass term is completely negligible, $m \ll H_{1}$, 
and all modes are relativistic. As the proper momentum is red-shifted, the modes become non-relativistic when $m$ $=k / a=\omega$, and re-enter the horizon when $H=\omega$.

For the axion field (4.14) the stochastic conditions (3.41) are still valid, but the squared amplitude (3.42), averaged over time scales $m / H \gg 1$, now become

$$
\begin{aligned}
\Sigma_{1}(\mathbf{p}, \eta) & =\frac{|c(\mathbf{k})|^{2}}{2 m a^{3}}\left(\frac{k}{k_{1}}\right)\left(\frac{H_{1}}{m}\right)^{1 / 2} \\
& =\frac{1}{m^{2} a^{2}} \Sigma_{2}(\mathbf{p}, \eta)=\frac{1}{m a} \Sigma_{3}(\mathbf{p}, \eta) .
\end{aligned}
$$

For the case we are considering, the contribution of $f_{\pi}$ to the Bardeen potentials is always negligible with respect to $\eta^{2} f_{\rho}$. An explicit computation gives, in fact,

$$
\eta^{2} f_{\rho} / f_{\pi} \simeq m / H \gg 1
$$

where the last inequality is a consequence of Eq. (4.13). In addition, the mass contribution to the AX energy density dominates with respect to the momentum contribution, since $m>k / a$. The energy density correlation function thus becomes

$$
\xi_{0}^{0}\left(x, x^{\prime}\right)=m^{4}\left(\left\langle\sigma^{2}(x) \sigma^{2}\left(x^{\prime}\right)\right\rangle-\left\langle\sigma^{2}(x)\right\rangle^{2}\right)
$$

(as $|\dot{\sigma}(k)|=m a|\sigma(k)|$ ), and gives, using Eq. (4.15):

$$
\begin{aligned}
k^{3}\left|f_{\rho}\right|^{2}\left(\frac{M}{a}\right)^{4}= & m^{4} k^{3} \int \frac{d^{3} p}{(2 \pi)^{3}} \Sigma_{1}(\mathbf{p}) \Sigma_{1}(\mathbf{k}-\mathbf{p}) \\
= & \frac{m H_{1}}{8 \pi^{2}}\left(\frac{k_{1}}{a}\right)^{6}\left(\frac{k}{k_{1}}\right)^{3} \\
& \times \int_{0}^{1} d y y^{2-2 \mu} \int_{-1}^{1} d x \beta^{-2 \mu}
\end{aligned}
$$

where $x, y$ and $\beta$ are defined in Sec. III B.

It should be noted that the above expression for the spectrum is only valid if $\mu>3 / 4$. Only in that case, in fact, is the integral over $y$ dominated by the contribution of the lower boundary, $p / k_{1} \rightarrow 0$, and is the use of Eq. (3.58) for the axion spectrum appropriate. In the opposite case, we have to take into account the different spectrum of non-relativistic subhorizon modes, for $p>k_{m}$, and possibly of relativistic modes in the high-frequency limit $p \rightarrow k_{1}$. In both cases we obtain, for $\mu<3 / 4$, a white noise spectrum and a negligible contribution to the large-scale anisotropy, as we will see in the next section.

We will thus concentrate on the case $3 / 4<\mu \leqslant 3 / 2$. For this case the integral (3.61) is estimated in Appendix B, and we obtain

$$
k^{3}\left|f_{\rho}\right|^{2}\left(\frac{M}{a}\right)^{4}=c_{m}^{2} m H_{1}\left(\frac{k_{1}}{a}\right)^{6}\left(\frac{k}{k_{1}}\right)^{6-4 \mu}, \quad 3 / 4<\mu \leqslant 3 / 2,
$$

where $c_{m}$ is a dimensionless number of order 1 . The corresponding Bardeen spectrum is

$$
\begin{aligned}
k^{3 / 2}|\Psi| & \sim k^{3 / 2}|\Phi| \sim \epsilon \eta^{2}\left|f_{\rho}\right| k^{3 / 2} \\
& =4 \pi G c_{m}(a \eta)^{2}\left(m H_{1}\right)^{1 / 2}\left(\frac{k_{1}}{a}\right)^{3}\left(\frac{k}{k_{1}}\right)^{3-2 \mu} \\
& \sim g_{1}^{2} c_{m}\left(\frac{m}{H_{1}}\right)^{1 / 2}\left(\frac{H_{1}}{H}\right)^{2}\left(\frac{a_{1}}{a}\right)^{3}\left(\frac{\omega}{\omega_{1}}\right)^{3-2 \mu} \\
& \sim \Omega_{\sigma}(\omega) .
\end{aligned}
$$

We may note that $\Psi H^{2}$ evolves in time like $a^{-3}$, so that, during the matter-dominated era (when $H^{2} \propto a^{-3}$ ), the Bardeen potential $\Psi$ remains frozen at the value reached at the time $\eta_{e q}$ of matter-radiation equilibrium. Using $\left(H_{1} / H_{e q}\right)^{2}\left(a_{1} / a_{e q}\right)^{3}=\left(a_{e q} / a_{1}\right)=\left(H_{1} / H_{e q}\right)^{1 / 2}$, we obtain for $\eta>\eta_{e q}$,

$$
k^{3 / 2}|\Psi| \sim k^{3 / 2}|\Phi| \sim c_{m} g_{1}^{2}\left(\frac{m}{H_{e q}}\right)^{1 / 2}\left(\frac{\omega}{\omega_{1}}\right)^{3-2 \mu} .
$$

The CMB anisotropy induced by the EM and AX seeds discussed here will be analyzed in the next section.

\section{CMB FLUCTUATIONS FROM PRE-BIG-BANG SEEDS}

For electromagnetic seeds, with the assumption that the electric field is already dissipated away at recombination, we find that the seeds are generically suppressed by a factor $\left(k \eta_{d e c}\right)^{2}$, and the anisotropic stress $f_{\pi}$ dominates over the density contribution $f_{\rho}$ (see the discussion at the end of Sec. II A). By contrast, for massless axionic perturbations, there is no $\left(k \eta_{d e c}\right)^{2}$ suppression for $f_{\rho}$, while there is one for $f_{\pi}$. For large wave numbers which enter the horizon before matter and radiation equality, EM and $\mathrm{AX}$ seeds lead to similar amplitudes. Consequently, if the convolution leading to $f_{\pi}$ is dominated by small scale contributions, $\mu<3 / 4$, the two cases give similar geometric scalar perturbations $\Psi, \Phi$, through Eq. (2.40).

However, on large scales, $k \eta_{e q}<1$, the additional axion production during the matter-dominated era leads to an enhancement by the factor $\left(\eta / \eta_{e q}\right)^{2}$. This changes the timedependence of the Bardeen potentials and has important consequences as we will see below.

\section{A. Electromagnetic seeds}

The scalar metric perturbation spectrum induced by EM seeds is reproduced in Eqs. (3.35) and (3.36). Comparing with our parametrization in terms of $\alpha$ and $\gamma$ [see Eqs. (2.59), (2.60)] we find

$$
\begin{aligned}
& \gamma= \begin{cases}-4, & \mu \leqslant 3 / 4 \\
2 \mu-11 / 2, & 3 / 4 \leqslant \mu \leqslant 3 / 2\end{cases} \\
& \alpha= \begin{cases}7 / 2, & \mu \leqslant 3 / 4 \\
5-2 \mu, & 3 / 4 \leqslant \mu \leqslant 3 / 2\end{cases}
\end{aligned}
$$

and 


$$
\mathcal{N}=c_{\pi}\left(\frac{g_{1}}{4 \pi}\right)^{2}\left(k_{1} \eta_{e q}\right)^{2}
$$

in both cases $\mu<3 / 4, \mu>3 / 4$ (modulo numbers of order 1 ).

Since $\gamma+1<0$, in both cases the seeds decay fast enough outside the horizon, and our analysis of Sec. II applies. However, in both cases $\gamma+\alpha=-0.5$, which leads to the spectral index $n=2$, i.e. to a spectrum that grows too fast with frequency to fit the results of COBE observations, see Eqs. (2.71), (2.72).

The quadrupole amplitude is given by $Q_{r m s}-P S$ $=\sqrt{(5 / 4 \pi) C_{2}} T_{0}$, which has been measured [34] to be $Q_{r m s-P S}=(18 \pm 2) \mu \mathrm{K}$. This leads to

$$
C_{2}=(1.09 \pm 0.23) \times 10^{-10} \text {. }
$$

From Eq. (2.70), using $\alpha+\gamma=-1 / 2, k_{1} \eta_{e q}=\left(H_{1} / H_{e q}\right)^{1 / 2}$, $g_{1}=H_{1} / M_{p}$, and setting $l=2$, we obtain:

$$
\begin{aligned}
C_{2}^{S W} \approx & \frac{c_{\pi}^{2} g_{1}^{6-\alpha}}{10(4 \pi)^{4}(\gamma+1)^{2}}\left(\frac{M_{p}}{H_{e q}}\right)^{2-\alpha} \\
& \times\left(\frac{\eta_{d e c}}{\eta_{0}}\right)^{2(\gamma+1)}\left(\frac{\eta_{e q}}{\eta_{0}}\right)^{2 \alpha}
\end{aligned}
$$

Compatibility with the COBE normalization, $C_{2} \lesssim 10^{-10}$, thus implies

$$
(6-\alpha) \log _{10} g_{1} \lesssim 55(\alpha-2)-6+\log _{10}(\gamma+1)^{2}-\log _{10} c_{\pi}^{2}
$$

(we have used $H_{e q} / M_{p} \sim 10^{-55}$, and $\eta_{d e c} \sim \eta_{e q} \sim 10^{-2} \eta_{0}$ ). This important constraint is easily satisfied by a growing seed spectrum, $\mu<3 / 2$, i.e. $\alpha>2$. In the limiting (and most unfavorable) case $\mu=3 / 2, \alpha=2, \gamma=-5 / 2$, the above condition reduces to

$$
\log _{10} g_{1} \lesssim-1.4-0.5 \log _{10} c_{\pi}
$$

Even in this limiting case there are no stringent constraints on the typical inflation scale of the "minimal" pre-big bang scenario $[1,7,8]$, expected to approach the string mass scale $M_{s}$ as $g_{1}=H_{1} / M_{p} \sim M_{s} / M_{p}$. Indeed, the limiting condition (4.7) is marginally compatible even with the maximal expected value $H_{1} \sim M_{s}$, since [35]

$$
10^{-2} \lesssim M_{s} / M_{p} \lesssim 10^{-1} \text {. }
$$

To conclude, the EM fluctuations seem to lead to a scalar perturbation spectrum that grows too fast with frequency to contribute in a significant way to the observed large-scale anisotropy. The positive aspect of our result is that there are no significant constraints from the COBE normalization to the production of seeds for galactic magnetic fields, which remains allowed as discussed in [11].

\section{B. Axionic seeds}

Let us first consider massless axions. If $\mu<3 / 4$, the situation is like in the electromagnetic case. The CMB fluctua- tions induced have the wrong spectrum, but their amplitude is sufficiently low to avoid conflict with observations.

If $3 / 4 \leqslant \mu \leqslant 3 / 2$ the situation becomes radically different. Comparing Eq. (3.53) with the ansatz (2.59), (2.60) we obtain, due to the additional factor $\left(\eta / \eta_{e q}\right)^{2}$,

$$
\begin{aligned}
& \gamma=2 \mu-7 / 2, \\
& \alpha=-\gamma-1 / 2=-2 \mu+3 .
\end{aligned}
$$

In the limiting case $\mu=3 / 2$ this yields $\gamma=-1 / 2$ and $\alpha=0$, which corresponds to a Harrison-Zel'dovich spectrum of CMB fluctuations, according to Eq. (2.73), with an amplitude

$$
\mathcal{N} \simeq g_{1}^{2}
$$

[we have absorbed into $g_{1}$ all dimensionless numerical coefficients of order one appearing in the spectrum (3.53)]. Note that $f_{\rho}$ leads to a Bardeen potential with the same $\alpha$, but with $\gamma=2 \mu-3 / 2$. However, since again $\gamma>-1$, the contribution to the SW effect is the same for $f_{\rho}$ and $f_{\pi}$ (see Sec. II).

The normalization of the axion spectrum to the COBE amplitude (4.4), according to Eq. (2.65), imposes the condition

$$
C_{2}^{S W} \simeq \mathcal{N}^{2}\left(k_{1} \eta_{0}\right)^{-2 \alpha} \simeq g_{1}^{4}\left(\frac{\omega_{0}}{\omega_{1}}\right)^{6-4 \mu} \simeq 10^{-10}
$$

which implies

$$
\log _{10} g_{1} \simeq \frac{164-116 \mu}{1+2 \mu}
$$

(again we have absorbed numerical coefficients into $g_{1}$, and we have used $\omega_{0} \sim 10^{-18} \mathrm{~Hz}, \omega_{1} \sim g_{1}^{1 / 2} 10^{11} \mathrm{~Hz}$, according to $[7,8])$. On the other hand, the allowed range for the spectral index [see Eq. (2.73)], combined with the condition $\mu \leqslant 3 / 2$ (required to prevent over-critical axion production), conservatively requiring $1 \leqslant n \leqslant 1.4$, implies

$$
1.4<\mu<1.5 \text {. }
$$

The combination of Eqs. (4.13), (4.14) leads to

$$
3 \times 10^{-3} \lesssim g_{1}=\left(H_{1} / M_{p}\right) \lesssim 2.6,
$$

which is perfectly compatible with the identification $H_{1}$ $\sim M_{s}$ [see Eq. (4.8)].

A stochastic background of massless axions, produced in the context of the pre-big-bang scenario, is thus a possible viable candidate for a consistent explanation of the largescale anisotropy observed by COBE. The important difference between AX and EM seeds is the non-conformal coupling of the axions to the metric, that leads to an additional amplification of perturbations after the matter-radiation equality.

Another interesting case is that of a massive axion background, for which the $f_{\pi}$ contribution to the Bardeen potentials is negligible when the super-horizon modes are already 
non-relativistic at the time of decoupling, $m>H_{d e c}$. As seen in the previous section, one then obtains constant Bardeen potentials, with $\gamma=0, \alpha=(n-1) / 2=3-2 \mu$ and

$$
\mathcal{N}=g_{1}^{2}\left(\frac{m}{H_{e q}}\right)^{1 / 2}
$$

[see Eq. (3.64), where we have set $c_{m}=1$ ]. A flat HarrisonZel'dovich spectrum is again possible in the limiting case $\mu=3 / 2$. The amplitude of perturbations, however, is enhanced by the factor $\left(m / H_{e q}\right)^{1 / 2}$, so that the value of the axion mass has to be bounded, to avoid conflicting with the COBE normalization (4.4).

We take again the allowed range for $\mu$ to be given by Eq. (4.14). In addition, the present axion energy density is constrained by the critical density bound, $\Omega_{\sigma}\left(\eta_{0}\right) \leqslant 1$, imposed at the peak frequency $\omega_{m}$ of non-relativistic modes (see Appendix C). Actually, an even stronger condition is required for the validity of our perturbative approach, which neglects the back-reaction of the axionic seeds on the expansion of the universe. Using Eq. (C25) we thus impose the condition

$$
\Omega_{\sigma}\left(\omega_{m}, \eta_{0}\right) \sim g_{1}^{2}\left(\frac{H_{1}}{H_{e q}}\right)^{1 / 2}\left(\frac{m}{H_{1}}\right)^{2-\mu} \leq 0.1
$$

which implies

$$
\begin{gathered}
(2-\mu)\left[\log _{10}\left(m / H_{e q}\right)-\log _{10} g_{1}-55\right] \\
+\frac{5}{2} \log _{10} g_{1}+\frac{55}{2}<-1 .
\end{gathered}
$$

In order to find a possible $\mathrm{AX}$ mass window compatible with the COBE data, we now impose the normalization $C_{2}$ $\simeq 10^{-10}$ on the massive axion spectrum Eq. (3.64). From Eq. (2.65) we obtain

$$
C_{2}^{S W} \simeq \mathcal{N}^{2}\left(k_{1} \eta_{0}\right)^{-2 \alpha} \simeq g_{1}^{4}\left(\frac{m}{H_{e q}}\right)\left(\frac{\omega_{0}}{\omega_{1}}\right)^{6-4 \mu} \simeq 10^{-10},
$$

from which

$$
\mu \simeq\left[164-\log _{10}\left(m / H_{e q}\right)-4 \log _{10} g_{1}\right] / 116
$$

(we have used $\omega_{1} / \omega_{0} \sim 10^{29}$, neglecting the weak dependence of $\omega_{1}$ on the transition scale $g_{1}$ ). By eliminating $\mu$ in terms of $m$ and $g_{1}$, according to the above equation, the constraints (4.14) and (4.18), plus the condition $m>H_{d e c}$ $\sim H_{e q}$ [assumed for the validity of the spectrum (3.64)], determine an allowed region in the plane $\left(m, H_{1}\right)$ as follows:

$$
\begin{aligned}
10^{-10}\left(M_{p} / H_{1}\right)^{4} & \leq m / H_{e q} \lesssim 10^{1.6}\left(M_{p} / H_{1}\right)^{4}, \quad m \gtrsim H_{e q}, \\
{[68} & \left.+\log _{10}\left(m / H_{e q}\right)+4 \log _{10} g_{1}\right] \\
& \times\left[\log _{10}\left(m / H_{e q}\right)-55-\log _{10} g_{1}\right] \\
& +58\left(55+5 \log _{10} g_{1}\right) \lesssim-1 .
\end{aligned}
$$

For a typical string cosmology scale, $H_{1} \sim M_{s} \sim\left(10^{-1}\right.$ $\left.-10^{-2}\right) M_{p}$, we thus obtain the maximal allowed window:

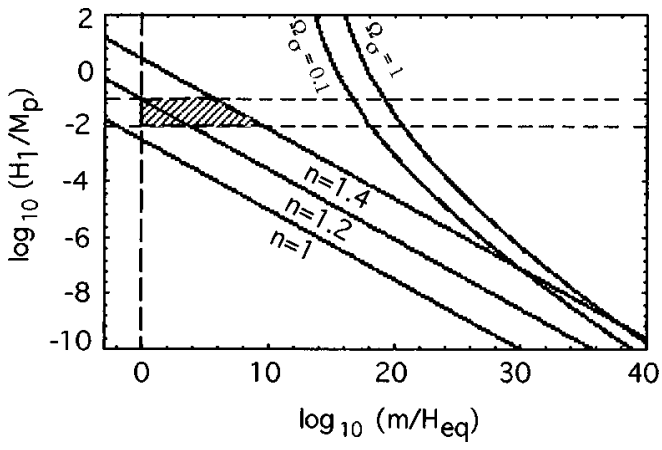

FIG. 1. The phenomenologically allowed region is to the left of the curve $\Omega_{\sigma}=0.1$, to the right of the vertical dashed line $m$ $=H_{e q}$, and lies within the full lines $n=1, n=1.4$, to avoid conflicting with present COBE observations ( $n<1$ is excluded by overcritical axion production). The shaded area defines the allowed mass window for an inflation scale $H_{1}=M_{s}$, typical of string cosmology.

$$
10^{-27} \mathrm{eV} \lesssim m \leqq 10^{-17} \mathrm{eV} \text {. }
$$

As illustrated in Fig. 1, the window is shifted towards higher values of mass as the final inflation scale is lowered and as the spectral index is increased. The seed condition (4.17) becomes important only at low inflation scales, $H_{1} / M_{p}$ $\lesssim 10^{-7}$. The stringent upper limit we obtained for the mass can be traced back to the simplest model of background used in this paper, that gives the same slope for the axion spectrum at low and high frequency [see Eqs. (C24), (C26)]. It is not excluded that higher values of the mass may become possible in a more complicated model of background, giving a steeper high frequency spectrum.

\section{CONCLUSIONS}

In this paper we have considered the possibility that, in a string cosmology context, the large-scale temperature anisotropies may arise from the contribution of seeds to the metric fluctuations, and not from the direct amplification of the metric fluctuations themselves, as in the conventional inflationary scenario. We have discussed, in particular, two cases: one in which the seeds are EM vacuum fluctuations amplified by the growth of the dilaton field, and one in which the seeds are AX vacuum fluctuations amplified by the time evolution of a higher-dimensional background.

In the case of EM perturbations we have found that the induced angular power spectrum of $\Delta T / T$ grows too fast to be compatible with COBE observations. However, the contribution of the seeds to the large-scale anisotropy may be consistently imposed to be negligible, without constraining in a significant way the basic parameters of the pre-big-bang models.

Massless AX perturbations, unlike EM perturbations, are also affected by the radiation $\rightarrow$ matter transitions. This changes the time dependence of the seed contribution to the Bardeen potentials and, due to the integrated Sachs-Wolfe effect, a flat or slightly tilted blue spectrum of temperature anisotropies can be induced, compatible with present COBE observations. Scale-invariant massless axion seeds thus ap- 
pear as possible promising candidates for structure formation. Determining in more details the CMB anisotropy spectrum also on smaller angular scales requires however numerical simulations, which we defer to a future research project.

For massive AX seeds the situation is qualitatively different if the mass is such that all modes outside the horizon at the time of decoupling are already non-relativistic. In that case the contribution to $\Delta T / T$ is controlled by the axion mass, and a slightly tilted blue spectrum is still compatible with the amplitude and the slope measured by COBE, provided the axion mass is inside an appropriate window, in the ultra-light mass region. Higher values of masses may become possible in models with more complicated backgrounds.

At smaller angular scales, an axionic origin of $\mathrm{CMB}$ anisotropies should lead to acoustic peaks in the spectrum, with a structure different from that of the standard inflationary scenario. This may in principle allow a test of models with axionic seeds through the very accurate observations of the CMB anisotropy planned in the near future [36]. It is possible that, in spite of the differences mentioned in the introduction, achieving enough power at scales smaller than COBE's will require a very blue spectrum $(n>1.5)$, as in the isocurvature CDM model discussed in Ref. [37]. A thorough investigation of this possibility is postponed to a future paper.

\section{ACKNOWLEDGMENTS}

We are grateful to Massimo Giovannini for helpful discussions. R.D. and M.S. are partially supported by the Swiss
NSF. M.S. acknowledges financial support from the Tomalla Foundation.

\section{APPENDIX A: SACHS-WOLFE COEFFICIENTS FOR POWER-LAW SPECTRA}

Assume that the Bardeen potentials are given by powerlaw spectra as in Eq. (2.58):

$$
\Psi-\Phi=\left\{\begin{array}{ll}
C(k) x^{\gamma}, & x \ll 1 \\
C(k), & x \gg 1,
\end{array} \quad C(k)=\mathcal{N} k^{-3 / 2}\left(k / k_{1}\right)^{\alpha},\right.
$$

where $x=k \eta, x_{0}=k \eta_{0}, x_{d e c}=k \eta_{d e c}$. The SW contribution to the angular coefficients $C_{l}$ is given by

$$
C_{l}^{S W}=\mathcal{N}^{2} \frac{2}{\pi} \int_{0}^{k_{1}} \frac{d k}{k}\left(\frac{k}{k_{1}}\right)^{2 \alpha}|I(k)|^{2}
$$

where

$$
I(k)=j_{l}\left(x_{0}-1\right)+\int_{x_{d e c}}^{1} x^{\gamma} j_{l}^{\prime}\left(x_{0}-x\right) d x,
$$

and a prime stands for the derivative of $j_{l}$ with respect to its argument.

We concentrate here on the case where $\gamma+1<0$. Furthermore, we are interested in the situation where the integral in Eq. (A2) is dominated by large scales (small values of $k$ ), and therefore $x_{d e c} \ll 1$. In that case the integral $I(k)$ is dominated by its value at the lower bound:

$$
I(k) \approx \frac{1}{|1+\gamma|} x_{d e c}^{\gamma+1} j_{l}^{\prime}\left(x_{0}\right)=\frac{1}{|1+\gamma|} x_{d e c}^{\gamma+1}\left[\frac{l}{2 l+1} j_{l-1}\left(x_{0}\right)-\frac{l+1}{2 l+1} j_{l+1}\left(x_{0}\right)\right] .
$$

This leads to the following expression for the $C_{l}$ 's:

$$
\begin{aligned}
C_{l}^{S W}= & \mathcal{N}^{2} \frac{2}{\pi}\left(\frac{\eta_{d e c}}{\eta_{0}}\right)^{2(\gamma+1)} \frac{1}{|1+\gamma|^{2}}\left(k_{1} \eta_{0}\right)^{-2 \alpha} \int_{0}^{\infty} \frac{d x_{0}}{x_{0}} x_{0}^{2(\alpha+\gamma+1)}\left[\frac{l^{2}}{(2 l+1)^{2}} j_{l-1}^{2}\left(x_{0}\right)-\frac{2 l(l+1)}{(2 l+1)^{2}} j_{l-1}\left(x_{0}\right) j_{l+1}\left(x_{0}\right)\right. \\
& \left.+\frac{(l+1)^{2}}{(2 l+1)^{2}} j_{l+1}^{2}\left(x_{0}\right)\right] \\
= & \frac{\mathcal{N}^{2}}{|1+\gamma|^{2}} \frac{2}{\pi}\left(\frac{\eta_{d e c}}{\eta_{0}}\right)^{2(\gamma+1)}\left(k_{1} \eta_{0}\right)^{-2 \alpha}\left[\frac{l^{2}}{(2 l+1)^{2}} I_{l}^{(1)}-\frac{2 l(l+1)}{(2 l+1)^{2}} I_{l}^{(2)}+\frac{(l+1)^{2}}{(2 l+1)^{2}} I_{l}^{(3)}\right],
\end{aligned}
$$

where, setting $j_{l}=\sqrt{\pi / x} J_{l-1 / 2}$, we find (Ref. [38], number 6.574), for $\alpha+\gamma<0$,

$$
\begin{aligned}
I_{l}^{(1)} & =\frac{\pi}{2} \int_{0}^{\infty} d x x^{2(\alpha+\gamma)} J_{l-1 / 2}^{2}(x) \\
& =\frac{\pi}{2} \frac{\Gamma(-2(\alpha+\gamma)) \Gamma(l+\alpha+\gamma)}{2^{-2(\alpha+\gamma)}[\Gamma(-(\alpha+\gamma)+1 / 2)]^{2} \Gamma(l-(\alpha+\gamma))} ;
\end{aligned}
$$




$$
\begin{aligned}
I_{l}^{(2)} & =\frac{\pi}{2} \int_{0}^{\infty} d x x^{2(\alpha+\gamma)} J_{l-1 / 2}(x) J_{l+3 / 2}(x) \\
& =\frac{\pi}{2} \frac{\Gamma(-2(\alpha+\gamma)) \Gamma(l+1+\alpha+\gamma)}{2^{-2(\alpha+\gamma)} \Gamma(-(\alpha+\gamma)-1 / 2) \Gamma(3 / 2-(\alpha+\gamma)) \Gamma(l+1-(\alpha+\gamma))} \\
I_{l}^{(3)} & =\frac{\pi}{2} \int_{0}^{\infty} d x x^{2(\alpha+\gamma)} J_{l+3 / 2}^{2}(x) \\
& =\frac{\pi}{2} \frac{\Gamma(-2(\alpha+\gamma)) \Gamma(l+2+\alpha+\gamma)}{2^{-2(\alpha+\gamma)}[\Gamma(-(\alpha+\gamma)+1 / 2)]^{2} \Gamma(l+2-(\alpha+\gamma))} .
\end{aligned}
$$

Finally, combining the above results, we obtain the result given in Eq. (2.70):

$$
\begin{aligned}
C_{l}^{S W}= & \frac{\mathcal{N}^{2}}{2^{-2(\alpha+\gamma)}(\gamma+1)^{2}} \frac{\Gamma(-2(\alpha+\gamma))}{\Gamma(1 / 2-\alpha-\gamma)^{2}}\left(\frac{\eta_{d e c}}{\eta_{0}}\right)^{2(\gamma+1)}\left(k_{1} \eta_{0}\right)^{-2 \alpha} \frac{\Gamma(l+1+\alpha+\gamma)}{\Gamma(l+1 \alpha-\gamma)} \\
& \times\left[\frac{l^{2}}{(2 l+1)^{2}} \frac{l-\alpha-\gamma}{l+\alpha+\gamma}+\frac{2 l(l+1)}{(2 l+1)^{2}} \frac{1 / 2+\alpha+\gamma}{1 / 2-\alpha-\gamma}+\frac{(l+1)^{2}}{(2 l+1)^{2}} \frac{l+1+\alpha+\gamma}{l+1-\alpha-\gamma}\right] .
\end{aligned}
$$

It is interesting to note that, for $\alpha+\gamma=-1 / 2$, the mixed term $I_{l}^{(2)}$ vanishes, which is indeed what happens in the case of electromagnetic seeds (see Sec. IV).

\section{APPENDIX B: THE SEED FUNCTIONS}

\section{Electromagnetic seeds}

For purely magnetic seeds, all the seed functions can be approximately determined by the energy density correlation function $\xi_{0}^{0}$, which leads to Eq. (3.30). The contribution of super-horizon modes $(k \eta \ll 1)$ to the spectrum can be estimated in the limit $z=k / k_{1} \rightarrow 0$. In this limit $\beta \rightarrow y, \cos ^{2} \alpha$ $\rightarrow 1$, and the integral (3.30) reduces to

$$
I=\frac{k^{3} k_{1}^{5}}{a^{8}} \int_{0}^{1} d y y^{2-4 \mu} \sin ^{4}\left(y k_{1} \eta\right), \quad \mu \leqslant 3 / 2 .
$$

The dominant region of integration is easily shown to be $y$ $\sim 1$ for $\mu \leqslant 3 / 4$ and $y k_{1} \eta \sim 1$ for $3 / 4 \leqslant \mu \leqslant 3 / 2$. This gives

$$
I= \begin{cases}\left(k_{1} / a\right)^{8}\left(k / k_{1}\right)^{3}, & \mu \leqslant 3 / 4, \\ \left(k_{1} / a\right)^{8}\left(k / k_{1}\right)^{3}\left(k_{1} \eta\right)^{4 \mu-3}, & 3 / 4 \leqslant \mu \leqslant 3 / 2,\end{cases}
$$

modulo numerical factors of order one. This coincides with the result reported in Eq. (3.32).

\section{Massless axions}

For massless axions, the seed spectral functions are determined by the integrals (3.46), (3.50). The various terms appearing in the integrands turn out to give comparable contributions, so let us concentrate on the typical term

$$
I=\frac{k^{3}}{a^{4}} \int d^{3} p p^{2}|\mathbf{k}-\mathbf{p}|^{2} \Sigma_{1}(\mathbf{p}) \Sigma_{1}(\mathbf{k}-\mathbf{p}) .
$$

We distinguish different integration regions: $0<p<k, k$ $<p<\eta^{-1}, \eta^{-1}<p<k_{e q}, k_{e q}<p<k_{1}$. The dominant integration regions depend on the value of $\mu$ but, for all $\mu$ $\leqslant 3 / 2$, they always lie at $p \geqslant \eta^{-1}>k$. This is the reason why we always obtain a white noise spectrum. On the other hand, the behavior in $\eta$ depends on which region of $p$ dominates. Specifically we find:

(1) For $3 / 4 \leqslant \mu \leqslant 3 / 2$ the leading contribution to $I$ comes from $p \sim \eta^{-1}$, and gives the single term appearing in Eq. (3.47).

(2) For $\mu<3 / 4$ the leading contribution comes either from $p \sim k_{1}$ [giving the first term in the square brackets of Eq. (3.47)], or (for $\mu$ very close to 3/4) from $p \sim \eta^{-1}$ (giving the second term in the same brackets).

\section{Massive axions}

For massive actions, the energy density spectrum is determined by Eq. (3.61), with $3 / 4<\mu \leqslant 3 / 2$. This integral is dominated by the region $p \sim k$, and its rough behavior can be easily obtained this way. For a more precise evaluation we proceed as follows: the angular integration gives

$$
\begin{aligned}
k^{3}\left|f_{\rho}\right|^{2}\left(\frac{M}{a}\right)^{4}= & \frac{m H_{1}}{16 \pi^{2} z(\mu-1)}\left(\frac{k_{1}}{a}\right)^{6}\left(\frac{k}{k_{1}}\right)^{3} \\
& \times \int_{0}^{1} d y y^{1-2 \mu}\left[(z-y)^{2-2 \mu}-(z+y)^{2-2 \mu}\right] .
\end{aligned}
$$

Defining $t=y / z$ we obtain

$$
\begin{aligned}
k^{3}\left|f_{\rho}\right|^{2}\left(\frac{M}{a}\right)^{4}= & \frac{m H_{1}}{16 \pi^{2}(\mu-1)}\left(\frac{k_{1}}{a}\right)^{6}\left(\frac{k}{k_{1}}\right)^{3} \\
& \times z^{3-4 \mu}(A-B)
\end{aligned}
$$


where, after some manipulation [38],

$$
\begin{aligned}
A & =\int_{0}^{\infty} d t t^{1-2 \mu}\left[(1-t)^{2-2 \mu}-(1+t)^{2-2 \mu}\right] \\
& =\frac{2^{4 \mu-4}}{\sqrt{\pi}} \Gamma(2-2 \mu) \Gamma(2 \mu-3 / 4)[\cos 2 \pi(\mu-1)-1]
\end{aligned}
$$

and

$$
B=\int_{1 / z}^{\infty} d t t^{1-2 \mu}\left[(1-t)^{2-2 \mu}-(1+t)^{2-2 \mu}\right]
$$

By evaluating this second integral in the limit $z \rightarrow 0$, we obtain

$$
B \sim z^{4 \mu-3} \ll A,
$$

so that

$$
\begin{aligned}
k^{3}\left|f_{\rho}\right|^{2}\left(\frac{M}{a}\right)^{4}=\frac{m H_{1} A}{16 \pi^{2}(\mu-1)}\left(\frac{k_{1}}{a}\right)^{6}\left(\frac{k}{k_{1}}\right)^{6-4 \mu}, & 3 / 4<\mu<3 / 2,
\end{aligned}
$$

as reported in Eq. (3.62). Note that there is no singularity for $\mu=1$, as

$$
\begin{aligned}
\lim _{\mu \rightarrow 1} & \frac{\Gamma(2-2 \mu)}{(\mu-1)}[\cos 2 \pi(\mu-1)-1] \\
= & \frac{4 \pi^{2}}{(\mu-1)^{2}}(\mu-1)^{2}=\text { const. }
\end{aligned}
$$

\section{APPENDIX C: NON-RELATIVISTIC CORRECTIONS TO THE AXION SPECTRUM}

For a massive-axion perturbation $\sigma$, the string frame action

$$
S=\frac{1}{2} \int d^{4} x \sqrt{-g} e^{\phi}\left[\left(\partial_{\mu} \sigma\right)^{2}-m^{2} \sigma^{2}\right],
$$

in a conformally flat background, can be written in terms of the canonical variable

$$
\psi=z \sigma, \quad z=a e^{\phi / 2},
$$

as

$$
S=\frac{1}{2} \int d^{3} x d \eta\left[\dot{\psi}^{2}-\left(\partial_{i} \psi\right)^{2}+\frac{\ddot{z}}{z} \psi^{2}-m^{2} a^{2} \psi^{2}\right]
$$

(the dot denotes differentiation with respect to the conformal time $\eta$ ). The Fourier modes $\psi_{k}$ satisfy the perturbation equation

$$
\ddot{\psi}_{k}+\left(k^{2}-\frac{\ddot{z}}{z}+m^{2} a^{2}\right) \psi_{k}=0 .
$$

We shall consider the background transition at $\eta=\eta_{1}$ from an initial pre-big-bang phase in which the axion is massless, to a final radiation-dominated phase in which the dilaton freezes to its present value, and the axion acquires a small (in string units) mass. For $\eta>\eta_{1}$ the solution of Eq. (C4) depends on the kinematics of the pump field $z$ and, after normalization to an initial vacuum spectrum, it can be written in terms of the second-kindHankel functions [30] as:

$$
\psi_{k}(\eta)=\eta^{1 / 2} H_{\mu}^{(2)}(k \eta) .
$$

In the radiation era, $\eta>\eta_{1}$, the "effective potential" $\ddot{z} / z$ is vanishing, and the perturbation equation reduces to

$$
\ddot{\psi}_{k}+\left(k^{2}+\alpha^{2} \eta^{2}\right) \psi_{k}=0,
$$

where we have put

$$
m^{2} a^{2}=\alpha^{2} \eta^{2}, \quad \alpha=m H_{1} a_{1}^{2},
$$

using the time behavior of the scale factor, $a \sim \eta$.

Assuming that the mass term is negligible at the transition scale, $m \ll k / a$, we can match the solution (C5) to the planewave solution

$$
\psi_{k}=\frac{1}{\sqrt{k}}\left[c_{+}(k) e^{-i k \eta}+c_{-}(k) e^{i k \eta}\right],
$$

and obtain:

$$
c_{ \pm}= \pm c(k) e^{ \pm i k \eta}, \quad|c(k)| \sim\left(k / k_{1}\right)^{-\mu-1 / 2} .
$$

(We are neglecting, for simplicity, numerical factors of order 1 , which are not very significant in view of the many approximations performed. Their contribution will be included into an overall numerical coefficient in front of the final spectrum.) In the relativistic regime, the amplified axion perturbation then takes the form:

$$
\sigma(\mathbf{k}, \eta)=\frac{c(\mathbf{k})}{a \sqrt{k}} \sin (k \eta),
$$

used in Sec. III C for the massless-axion case.

In the radiation era the proper momentum is red-shifted with respect to the rest mass, and all axion modes tend to become non-relativistic. When the mass term is no longer negligible, the general solution of Eq. (C6) can be written in terms of parabolic cylinder functions [30]. For an approximate estimate of the axion field in the non-relativistic regime, however, it is convenient to distinguish two cases, depending on whether a mode $k$ becomes non-relativistic inside or outside the horizon. Defining as $k_{m}$ the limiting comoving frequency of a mode that becomes non-relativistic $\left(k_{m}\right.$ $\left.=m a_{m}\right)$ at the time it re-enters the horizon $\left(k_{m}=H_{m} a_{m}\right)$, we find, in the radiation era,

$$
k_{m}=k_{1}\left(\frac{m}{H_{1}}\right)^{1 / 2} .
$$

We will thus consider the two cases $k \gg k_{m}$ and $k \ll k_{m}$. 
In the first case, we rewrite the perturbation equation (C6) as

$$
\frac{d^{2} \psi_{k}}{d x^{2}}+\left(\frac{x^{2}}{4}-b\right) \psi_{k}=0, \quad x=\eta(2 \alpha)^{1 / 2}, \quad-b=k^{2} / 2 \alpha,
$$

and we give the general solution in the form

$$
\psi=A W(b, x)+B W(b,-x),
$$

where $W(b, x)$ are the Weber parabolic cylinder functions (see [30], Chap. 19). In order to fix the integration constants $A$ and $B$ we shall match the solutions $(\mathrm{C} 13)$ and $(\mathrm{C} 10)$ in the relativistic limit

$$
\frac{k^{2}}{m^{2} a^{2}}=\frac{k^{2}}{\alpha^{2} \eta^{2}}=\frac{-4 b}{x^{2}} \gg 1
$$

In this limit, as we are considering modes that become nonrelativistic when they are already inside the horizon,

$$
\left(\frac{k}{k_{m}}\right)^{2} \sim \frac{k^{2}}{\alpha} \sim(-b) \gg 1,
$$

we can expand the $W$ functions for $b$ large with $x$ moderate [30]. Matching to the plane-wave solution (C10), we obtain $A=0$, and

$$
\psi_{k} \simeq \frac{c(\mathbf{k})}{\alpha^{1 / 4}} W(b,-x)
$$

In the opposite, non-relativistic limit $x^{2} \gg|4 b|$, the expansion of the Weber functions gives [30]

$$
\psi_{k} \simeq \frac{c(\mathbf{k})}{(\alpha \eta)^{1 / 2}} \sin \left(\frac{m}{H}\right)
$$

(we have used $x^{2} / 4=\operatorname{ma\eta } / 2 \sim m / H$ ). The corresponding axion field is (inside the horizon)

$$
\sigma(\mathbf{k}, \eta)=\frac{c(\mathbf{k})}{a \sqrt{m a}} \sin \left(\frac{m}{H}\right), \quad k>k_{m} .
$$

Consider now the case of a mode that becomes nonrelativistic when it is still outside the horizon, $k \ll k_{m}$. In this case, we cannot use the large $|b|$ expansion as $|b|<1$, and it is convenient to express the general solution of Eq. (C12) as

$$
\psi=A y_{1}(b, x)+B y_{2}(b, x),
$$

where $y_{1}$ and $y_{2}$ are the even and odd parts of the parabolic cylinder functions [30]. Matching to Eq. (C10), in the relativistic limit $x \rightarrow 0$, gives $A=0$ and

$$
\psi_{k} \simeq c(\mathbf{k})\left(\frac{k}{2 \alpha}\right)^{1 / 2} y_{2}(b, x)
$$

In the non-relativistic limit $x^{2} \gg|b|$ we use the relation [30]

$$
y_{2} \sim[W(b, x)-W(b,-x)] \sim \frac{1}{\sqrt{x}} \sin \frac{x^{2}}{4},
$$

which leads to

$$
\psi_{k} \simeq \frac{c(\mathbf{k})}{(\alpha \eta)^{1 / 2}}\left(\frac{k^{2}}{\alpha}\right)^{1 / 4} \sin \left(\frac{m}{H}\right) .
$$

Using Eqs. (C15) and (C11) for $k^{2} / \alpha$, we finally arrive at the non-relativistic axion field presented in Eq. (4.14):

$$
\sigma(\mathbf{k}, \eta)=\frac{c(\mathbf{k})}{a \sqrt{m a}}\left(\frac{k}{k_{1}}\right)^{1 / 2}\left(\frac{H_{1}}{m}\right)^{1 / 4} \sin \left(\frac{m}{H}\right), \quad k<k_{m} .
$$

For later use, it is also convenient to define the spectral energy density in critical units, $\Omega_{\sigma}(\omega)=d\left(\rho / \rho_{c}\right) / d \ln \omega$, associated with the stochastic axion background in the three different regimes defined before.

For relativistic modes we find, from Eq. (C10),

$$
\Omega_{\sigma}(\omega) \sim g_{1}^{2}\left(\frac{\omega}{\omega_{1}}\right)^{3-2 \mu}\left(\frac{H_{1}}{H}\right)^{2}\left(\frac{a_{1}}{a}\right)^{4}, \quad m<\omega<\omega_{1} .
$$

For modes that becomes non-relativistic after re-entry we find, from Eq. (C18),

$$
\Omega_{\sigma}(\omega) \sim g_{1}^{2} \frac{m}{H_{1}}\left(\frac{\omega}{\omega_{1}}\right)^{2-2 \mu}\left(\frac{H_{1}}{H}\right)^{2}\left(\frac{a_{1}}{a}\right)^{3}, \quad \omega_{m}<\omega<m .
$$

For modes that becomes non-relativistic before re-entry we find, from Eq. (C23),

$$
\Omega_{\sigma}(\omega) \sim g_{1}^{2}\left(\frac{m}{H_{1}}\right)^{1 / 2}\left(\frac{\omega}{\omega_{1}}\right)^{3-2 \mu}\left(\frac{H_{1}}{H}\right)^{2}\left(\frac{a_{1}}{a}\right)^{3}, \quad \omega<\omega_{m} .
$$

The last two spectral distributions are constant during the matter-dominated era, and the last one corresponds to the spectrum of the Bardeen potentials, as given in Eq. (3.63).
[1] G. Veneziano, Phys. Lett. B 265, 287 (1991); M. Gasperini and G. Veneziano, Astropart. Phys. 1, 317 (1993); Mod. Phys. Lett. A 8, 3701 (1993); Phys. Rev. D 50, 2519 (1994). An updated collection of papers on the pre-big-bang scenario is available at http://www.to.infn.it/ gasperin/.

[2] R. Brandenberger and C. Vafa, Nucl. Phys. B316, 391 (1989); Veneziano [1]; A. A. Tseytlin, Mod. Phys. Lett. A 6, 1721 (1991); K. A. Meissner and G. Veneziano, Phys. Lett. B 267,
33 (1991); Mod. Phys. Lett. A 6, 3397 (1991); A. Sen, Phys. Lett. B 271, 295 (1991); S. F. Hassan and A. Sen, Nucl. Phys. B375, 103 (1992); A. A. Tseytlin and C. Vafa, ibid. B372, 443 (1992); M. Gasperini and G. Veneziano, Phys. Lett. B 277, 256 (1992).

[3] G. Veneziano, Phys. Lett. B 406, 297 (1997); A. Buonanno et al., Phys. Rev. D 57, 2543 (1998).

[4] R. Brustein and G. Veneziano, Phys. Lett. B 329, 429 (1994); 
N. Kaloper, R. Madden, and K. A. Olive, Nucl. Phys. B452, 677 (1995); Phys. Lett. B 371, 34 (1996); R. Easther, K. Maeda, and D. Wands, Phys. Rev. D 53, 4247 (1996); R. Easther and K. Maeda, ibid. 54, 7252 (1996); M. Gasperini, J. Maharana, and G. Veneziano, Nucl. Phys. B472, 349 (1996); S. J. Rey, Phys. Rev. Lett. 77, 1929 (1996); M. Gasperini and G. Veneziano, Phys. Lett. B 387, 715 (1996); M. Gasperini, M. Maggiore, and G. Veneziano, Nucl. Phys. B494, 315 (1997); R. Brustein and R. Madden, Phys. Lett. B 410, 110 (1997); Phys. Rev. D 57, 712 (1988).

[5] M. S. Turner and E. J. Weinberg, Phys. Rev. D 56, 4604 (1997); M. Maggiore and R. Sturani, Phys. Lett. B 415, 335 (1997); N. Kaloper, A. Linde, and R. Bousso, Phys. Rev. D (to be published), hep-th/9801073.

[6] G. F. Smoot et al., Astrophys. J. Lett. 396, L1 (1992); C. L. Bennet et al., Astrophys. J. 430, 423 (1994).

[7] M. Gasperini and M. Giovannini, Phys. Lett. B 282, 36 (1991); Phys. Rev. D 47, 1519 (1993); Gasperini and Veneziano [1]; R. Brustein, M. Gasperini, M. Giovannini, V. F. Mukhanov, and G. Veneziano, Phys. Rev. D 51, 6744 (1995).

[8] R. Brustein, M. Gasperini, M. Giovannini, and G. Veneziano, Phys. Lett. B 361, 45 (1995); R. Brustein, M. Gasperini, and G. Veneziano, Phys. Rev. D 55, 3882 (1997).

[9] M. Gasperini, Phys. Rev. D 56, 4815 (1997).

[10] R. Durrer, Phys. Rev. D 42, 2533 (1990).

[11] M. Gasperini, M. Giovannini, and G. Veneziano, Phys. Rev. Lett. 75, 3796 (1995); D. Lemoine and M. Lemoine, Phys. Rev. D 52, 1955 (1995).

[12] E. J. Copeland, R. Easther, and D. Wands, Phys. Rev. D 56, 874 (1997); E. J. Copeland, J. E. Lidsey, and D. Wands, Nucl. Phys. B56, 506 (1997).

[13] M. Gasperini, M. Giovannini, and G. Veneziano, Phys. Rev. D 52, 6651 (1995).

[14] R. Durrer and M. Sakellariadou, Phys. Rev. D 56, 4480 (1997).

[15] R. K. Sachs and A. M. Wolfe, Astrophys. J. 147, 73 (1967).

[16] M. Axenides, R. H. Brandenberger, and M. S. Turner, Phys. Lett. 126B, 178 (1983).
[17] M. Kawasaki, N. Sugiyama, and T. Tanagida, Phys. Rev. D 54, 2442 (1996).

[18] R. Durrer, Fundam. Cosm. Phys. 15, 209 (1994).

[19] J. Bardeen, Phys. Rev. D 22, 1882 (1980).

[20] H. Kodama and M. Sasaki, Prog. Theor. Phys. Suppl. 78, 1 (1984).

[21] R. Durrer and N. Straumann, Helv. Phys. Acta 61, 1027 (1988).

[22] J. M. Steward and M. Walker, Proc. R. Soc. London A341, 49 (1974).

[23] R. Durrer, A. Gangui, and M. Sakellariadou, Phys. Rev. Lett. 76, 579 (1996).

[24] R. Bond and G. Efstathiou, Mon. Not. R. Astron. Soc. 226, 655 (1987).

[25] C. Bennett et al., Astrophys. J. Lett. 464, L1 (1996).

[26] H. R. Harrison, Phys. Rev. D 1, 2726 (1970); Y. B. Zel'dovich, Mon. Not. R. Astron. Soc. 160, 1 (1972).

[27] R. R. Metsaev and A. A. Tseytlin, Nucl. Phys. B293, 385 (1987).

[28] A. Buonanno, K. Meissner, C. Ungarelli, and G. Veneziano, J. High Energy Phys. 01, 004 (1998).

[29] V. F. Mukhanov, A. Feldman, and R. Brandenberger, Phys. Rep. 215, 203 (1992).

[30] M. Abramowitz and I. A. Stegun, Handbook of Mathematical Functions (Dover, New York, 1972).

[31] M. Gasperini, in Proceedings of the 12th International Conference on General Relativity and Gravitational Physics, Rome, 1996, edited by M. Bassan et al. (World Scientific, Singapore, 1997), p. 181.

[32] M. S. Turner and L. M. Widrow, Phys. Rev. D 37, 2743 (1988).

[33] M. Giovannini, Ph.D. thesis, Turin University, 1996.

[34] A. J. Banday et al., Astrophys. J. 475, 393 (1997).

[35] V. Kaplunovsky, Phys. Rev. Lett. 55, 1036 (1985).

[36] M. Bersanelli et al., "COBRAS/SAMBA, Report on the phase A study,', ESA Document D/SCI(96) 3 (1996).

[37] P. J. E. Peebles, reports archived under astro-ph/9805194 and astro-ph/9805212, 1998.

[38] I. S. Gradshteyn and I. M. Ryzhik, Table of Integrals, Series and Products (Academic, New York, 1965). 